\title{
Synthesis of 2-Indol-3-ylbenzofulvenes through a Tandem Reaction Catalyzed by Cationic Au(I) Complexes
}

\author{
Estela Álvarez, ${ }^{\mathrm{a}}$ Delia Miguel, ${ }^{\mathrm{a} 1}$ Patricia García-García, ${ }^{\mathrm{a}}$ Manuel A. Fernández-Rodríguez, ${ }^{\mathrm{a}}$ Félix Rodríguez, \\ Roberto Sanz $*^{\mathrm{a}}$ \\ aÁrea de Química Orgánica, Departamento de Química, Facultad de Ciencias, Universidad de Burgos, Pza. Misael Bañuelos s/n, 09001- \\ Burgos, Spain \\ 'Instituto Universitario de Química Organometálica “Enrique Moles”, Universidad de Oviedo, C/ Julián Clavería, 8, 33006-Oviedo, \\ Spain \\ Fax: +(34)947258831 \\ E-mail: rsd@ubu.es
}

Received: The date will be inserted once the manuscript is accepted. Dedicated to our good friend Professor Francisco J. Fañanás on the occasion of his $60^{\text {th }}$ birthday

\begin{abstract}
A new access to benzofulvenes bearing an indol-3-yl substituent at C-2 has been developed. Treatment of 3propargylindoles possessing an additional hydroxyl group at the other propargylic position with a cationic gold(I) complex triggers a tandem 1,2-indole migration / aura-iso-Nazarov cyclization / elimination sequence that takes place under mild conditions.
\end{abstract}

Key words: benzofulvenes, catalysis, gold, indoles, tandem reactions

The terms domino, cascade, or tandem reactions are generally used to designate a chemical transformation of an organic molecule through two or more consecutive elaborations with a single workup step. ${ }^{2}$ Several authors have distinguished between domino or cascade reactions in which the starting material undergoes a transformation via two (domino) or more (cascade) reactions one after another in an inseparable fashion, ${ }^{3}$ and tandem reactions that are considered to be two-step reactions that proceed in a consecutive fashion where each of the steps can be performed separately. ${ }^{4}$ So in the case of cascade catalysis, intermediates are not generally isolable and multiple transformations take place via a single catalytic mechanism. Regardless of the notation of this type of reactions, these are of great interest because they offer a convenient and economical manner to access to complex organic molecules in general from simple starting materials in a one-pot process with all the catalysts being present from the beginning.

Whereas most of the catalyzed cascade or tandem reactions have been triggered by palladium catalysts, ${ }^{6}$ in the last years several examples of these processes (often denoted as tandem) have been reported in the context of gold catalysis. ${ }^{7}$ Moreover, many of these tandem reactions are initiated by acyloxy migrations. ${ }^{8}$

On the other hand, benzofulvenes are interesting compounds that have found versatile applications in material science, ${ }^{9}$ as precursors of indenyl ligands ${ }^{10}$ and other indene derivatives, ${ }^{11}$ and in medicinal chemistry. $^{12}$ So, different approaches to methyleneindenes are known, mainly from indane or indanone derivatives, although more recently some useful routes have been developed based on the direct cyclization of monocyclic precursors. ${ }^{13}$
We have recently studied the behavior of 3propargylindoles, easily available by direct nucleophilic substitution of propargylic alkynols with indoles, ${ }^{14}$ in the presence of cationic gold complexes, and we have described that the indole nucleus is able to undergo a 1,2-migration process triggering different tandem reactions depending on the substitution pattern of the starting alkyne. ${ }^{15}$ When an (hetero)aromatic substituent is present at the propargylic position of the starting 3-propargylindole, a metalla-iso-Nazarov reaction follows the initial 1,2-migration of the indole. ${ }^{16}$ This tandem sequence affords interesting 2(indol-3-yl)indene derivatives with a wide variety of different substituents at C-1 (Scheme 1). ${ }^{15 b}$

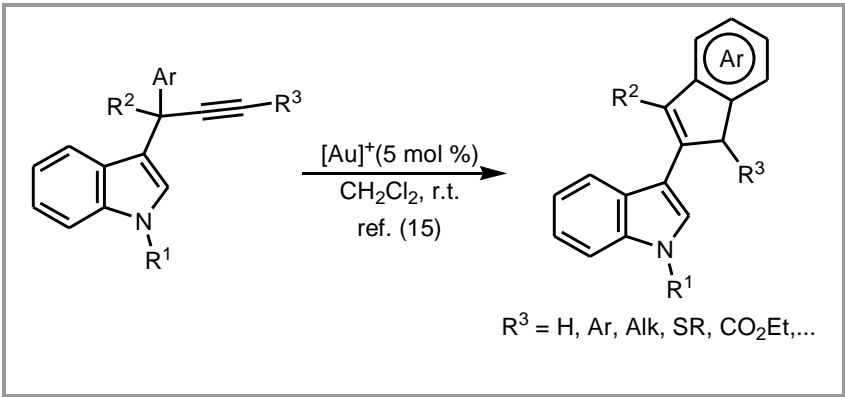

Scheme 1 Gold(I)-Catalyzed Tandem 1,2-Indole Migration / IsoNazarov Reactions of 3-Propargylindoles

On the other hand, it is well-known that propargylic esters are able to undergo 1,2- or 1,3-acyloxy migration under gold-catalysis, giving rise to diverse products depending on the subsequent evolution of the initially formed Au-carbenoid or Au-allene complex intermediate. ${ }^{17}$ So, at this point and following our interest in the synthesis of indene derivatives, ${ }^{\text {I }}$ we decided to evaluate the reactivity of an internal alkyne containing an indole at one of the propargylic positions and an acetate group at the other propargylic position. So, in a starting material like this one, a 1,2indole- or a 1,2-acetate-migration could be the initial step of a tandem process.

With this aim compound 1a was treated with a catalytic amount of the gold complex $\mathrm{Ph}_{3} \mathrm{PAuNTf}_{2}$, developed by Gagosz and co-workers, ${ }^{19}$ in 
dichloromethane at room temperature. Under these conditions product $\mathbf{3 a}$, arising from the previously reported tandem 1,2-indole migration / aura-isoNazarov cyclization reaction, was obtained in $31 \%$ yield as a mixture of diastereoisomers. Moreover, a new product $\mathbf{5 a}$, possessing an interesting benzofulvene core, was also isolated in $57 \%$ yield as a ca. 2 / 1 mixture of geometrical isomers (Scheme 2). The structure of both compounds, 3a and 5a, indicates that the migration of the indole has selectively occurred without competitive acetate migration. In addition, starting from alkynol 2a instead of acetate 1a, alcohol 4a was obtained as a mixture of diastereoisomers $(36 \%$ yield $), \quad$ whereas methyleneindene 5a was isolated in $41 \%$ yield and as a single geometrical isomer (Scheme 2). ${ }^{20}$

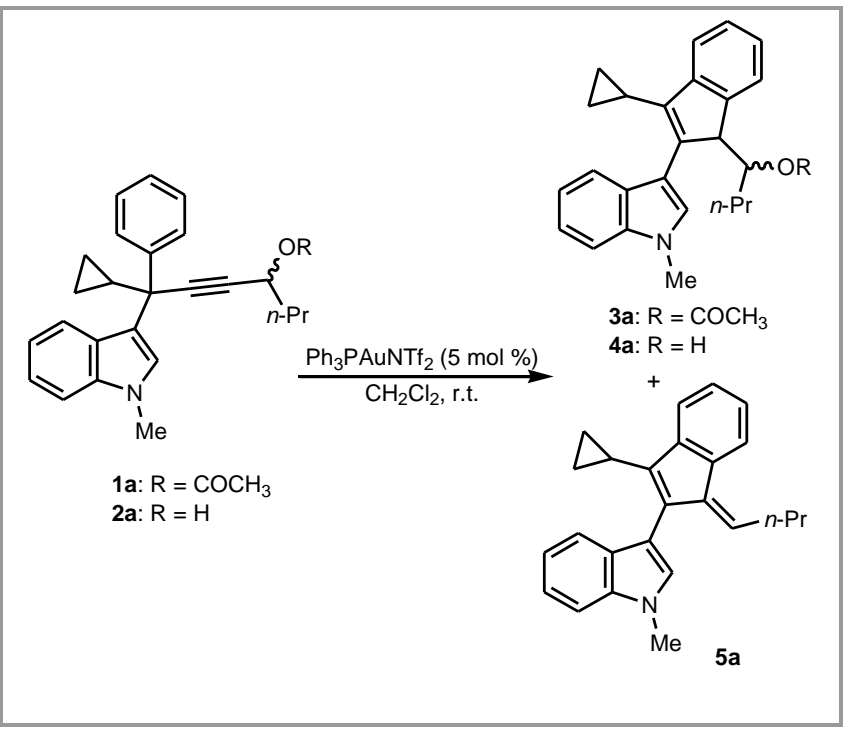

Scheme 2 Reaction of Propargylic Acetate 1a and Alkynol 2a under Gold(I)-Catalysis

Although benzofulvene 5a could be thought to be produced from a simple elimination reaction under the reaction conditions from the corresponding acetate $\mathbf{3 a}$ or alcohol 4a, we ruled out this possibility by checking that isolated $\mathbf{4 a}$ did not afford $\mathbf{5 a}$ by its treatment with the catalyst under the reaction conditions or even at reflux. Due to the interest in the development of novel routes for the generation of functionalized 1-methyleneindenes, ${ }^{13}$ we decided to further investigate this tandem reaction with the aim of improving its selectivity towards the benzofulvene derivative $\mathbf{5 a}$.

For the screening experiments we selected alkynol $\mathbf{2 a}$ as a model substrate (Table 1). After the initial experiment at room temperature with $\mathrm{Ph}_{3} \mathrm{PAuNTf}_{2}$ (entry 1), the effect of an acid or base additive was checked and no change on the selectivity was observed (entries 2 and 3). Switching the $\mathrm{Ph}_{3} \mathrm{P}$ ligand to the more basic XPhos (entry 4) or $\mathrm{Et}_{3} \mathrm{P}$ (entry 5) gave rise to a minor amount of the desired benzofulvene. Gratifyingly, we could enhance the ratio of 5a / 4a by using a gold complex bearing the $\operatorname{IPr}$ (entry 6) as ligand. Finally, the employment of the less basic phosphite ligand afforded the highest ratio of 5a / 4a, ca. 3.5 / 1 (entry 7). Once the triphenylphosphite was selected as the best ligand to favor the desired tandem process, the influence of the solvent was studied. However, none of the tested solvents (entries 8-11) afforded a better result than that obtained with $\mathrm{CH}_{2} \mathrm{Cl}_{2}$ (entry 7). Then, different silver salts, used for the generation of the cationic gold catalyst, were tested (entries 12-15) and similar selectivities were observed with all of them showing that the gold(I) counterion has a negligible effect. Finally, with the optimized catalytic system, $(\mathrm{PhO})_{3} \mathrm{PAuCl} / \mathrm{AgNTf}_{2}$ in $\mathrm{CH}_{2} \mathrm{Cl}_{2}$, a further improvement on the selectivity was observed by lowering the temperature. Thus, carrying out the reaction at $-78{ }^{\circ} \mathrm{C}$ afforded a $7 / 1$ ratio in favor of the desired 1-methyleneindene $\mathbf{5 a}$, which was isolated as a single geometrical isomer in $75 \%$ yield (entry 16 ).

Table 1 Effect of the catalyst and reaction conditions on the reactivity of $\mathbf{2} \mathbf{a}^{\mathbf{a}}$
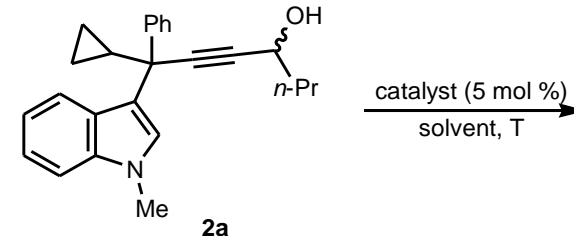


Solvent

$\mathrm{CH}_{2} \mathrm{Cl}_{2}$

$\mathrm{CH}_{2} \mathrm{Cl}_{2}$

$\mathrm{CH}_{2} \mathrm{Cl}_{2}$

$\mathrm{CH}_{2} \mathrm{Cl}_{2}$

$\mathrm{CH}_{2} \mathrm{Cl}_{2}$

$\mathrm{CH}_{2} \mathrm{Cl}_{2}$

$\mathrm{CH}_{2} \mathrm{Cl}_{2}$

toluene $\begin{array}{ll}(\mathrm{PhO})_{3} \mathrm{PAuCl} / \mathrm{AgNTf}_{2} & \mathrm{THF} \\ (\mathrm{PhO})_{3} \mathrm{PAuCl} / \mathrm{AgNTf}_{2} & \mathrm{DME}\end{array}$ $\begin{array}{ll}(\mathrm{PhO})_{3} \mathrm{PAuCl} / \mathrm{AgNTf}_{2} & \mathrm{THF} \\ (\mathrm{PhO})_{3} \mathrm{PAuCl} / \mathrm{AgNTf}_{2} & \mathrm{DME}\end{array}$

\section{Ratio 4a / 5a}

$1 / 2$

$1 / 2$

$1 / 2$

$2 / 1$

$1.5 / 1$

$1 / 3^{\mathrm{e}}$

$1 / 3.5$

$1 / 2$

$1 / 3^{\mathrm{e}}$ 


\begin{tabular}{|c|c|c|c|c|}
\hline 11 & $(\mathrm{PhO})_{3} \mathrm{PAuCl} / \mathrm{AgNTf}_{2}$ & $\mathrm{MeNO}_{2}$ & r.t. & $1 / 3$ \\
\hline 12 & $(\mathrm{PhO})_{3} \mathrm{PAuCl} / \mathrm{AgOTf}$ & $\mathrm{CH}_{2} \mathrm{Cl}_{2}$ & r.t. & $1 / 3$ \\
\hline 13 & $(\mathrm{PhO})_{3} \mathrm{PAuCl} / \mathrm{AgBF}_{4}$ & $\mathrm{CH}_{2} \mathrm{Cl}_{2}$ & r.t. & $1 / 3$ \\
\hline 14 & $(\mathrm{PhO})_{3} \mathrm{PAuCl} / \mathrm{AgOTs}$ & $\mathrm{CH}_{2} \mathrm{Cl}_{2}$ & r.t. & $1 / 3$ \\
\hline 15 & $(\mathrm{PhO})_{3} \mathrm{PAuCl} / \mathrm{AgSbF}_{6}$ & $\mathrm{CH}_{2} \mathrm{Cl}_{2}$ & r.t. & $1 / 3$ \\
\hline 16 & $(\mathrm{PhO})_{3} \mathrm{PAuCl} / \mathrm{AgNTf}_{2}$ & $\mathrm{CH}_{2} \mathrm{Cl}_{2}$ & -78 & $1 / 7$ \\
\hline
\end{tabular}

${ }^{\text {a }}$ All reactions were carried out with $0.1 \mathrm{mmol}$ of $\mathbf{2 a}$ in $0.2 \mathrm{~mL}$ of the corresponding solvent. Conversion was complete and the reactions exclusively afforded compounds $\mathbf{4 a}$ and $\mathbf{5 a}$.

${ }^{\mathrm{b}}$ Determined by ${ }^{1} \mathrm{H}$ NMR analysis of the crude reaction mixture.

${ }^{c}$ XPhos = 2-dicyclohexylphosphino-2',4',6'-triisopropylbiphenyl.

${ }^{\mathrm{d}} \operatorname{IPr}=1$,3-bis(2,6-diisopropylphenyl)imidazol-2-ylidene.

${ }^{\mathrm{e}}$ Benzofulvene 5a was obtained as a mixture of geometrical isomers.

In order to examine the scope of this tandem process we prepare, in first place, various alkynols 2 using our developed methodology for accessing 3propargylindoles (Scheme 3). ${ }^{14}$ Thus, terminal alkynes 8 obtained from $N$-methylindole 6 by PTSA-catalyzed direct substitution of terminal alkynols 7 , were reacted with $n$-BuLi and then with different carbonyl derivatives affording alkynols 2a-c,e-h in good yields (Scheme 3). On the other hand, other propargylic alcohols like 2d,i-l could be prepared in only one step from diols 9 by regioselective nucleophilic substitution of the more activated hydroxyl group with indole 6, under the same Brønsted acid-catalyzed conditions (Scheme 3). As expected, all these alkynols 2 were obtained as a ca. $1 / 1$ mixture of diastereoisomers.

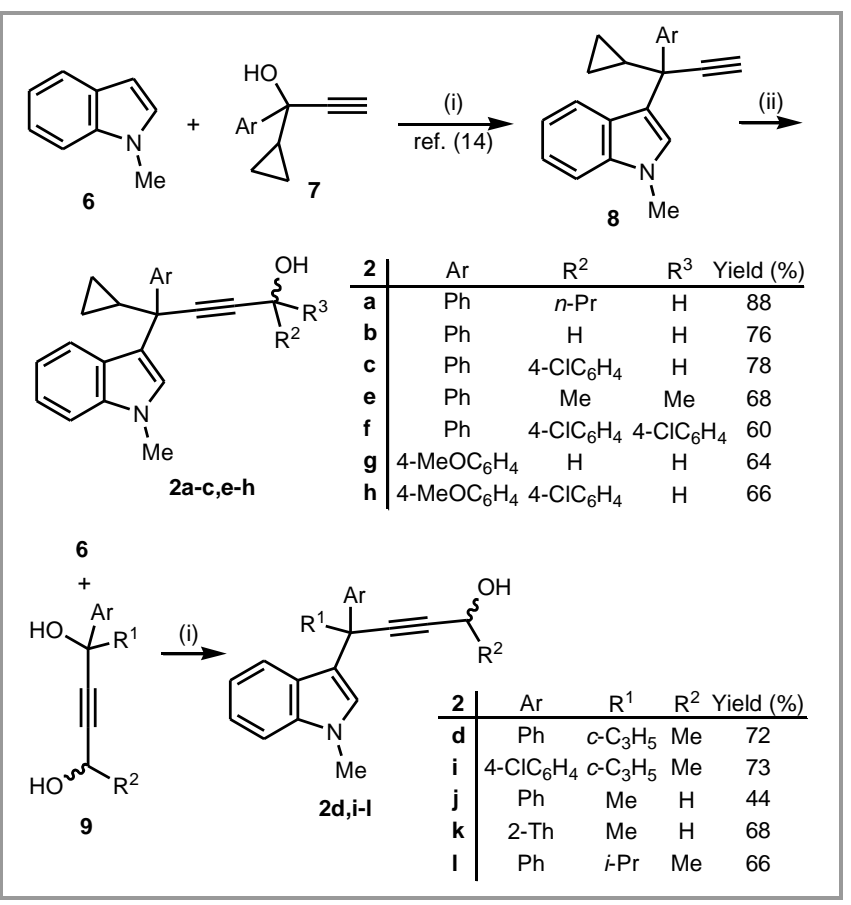

Scheme 3 Synthesis of Alkynols 2 from $N$-methylindole 6. Reagents and conditions: (i) PTSA (5 $\mathrm{mol} \%$ ), $\mathrm{MeCN}$, r.t.; (ii) (a) $n$-BuLi (1.1 equiv), THF, $-78{ }^{\circ} \mathrm{C}$ to r.t.; (b) $\mathrm{R}^{2} \mathrm{COR}^{3}$ (1.1 equiv), $-78{ }^{\circ} \mathrm{C}$ to r.t.
With alkynols 2 in hand, we evaluated the effect of the substitution pattern at the hydroxylic carbon (Table 2, entries 1-6). Surprisingly, we found that the methyleneindene derivative was also the major product when using the primary propargylic alcohol $\mathbf{2 b}$ and so, we isolated benzofulvene $\mathbf{5 b}$ in $\mathbf{7 4} \%$ yield (entry 2). A similar behaviour was observed in the case of a secondary benzylic alkynol such as $2 \mathrm{c}$ that affords benzofulvene $\mathbf{5 c}$ in excellent yield and as a single geometrical isomer, whose structure was confirmed by using single-crystal X-ray analysis (entry 3). ${ }^{21}$ Even in the case of alkynol $2 \mathbf{d}$ possessing a methyl group at the propargylic position adjacent to the hydroxyl $\left(\mathrm{R}^{2}\right)$, the corresponding benzofulvene $\mathbf{5 d}$ was obtained in high yield and with complete stereoselectivity (entry 4). However, with tertiary alkynols $2 \mathbf{e}$ and $\mathbf{2 f}$ the formation of the corresponding 1-tert-hydroxyalkylindenes $\mathbf{4 e}$ and $\mathbf{4 f}$ was preferred and when starting with alkynol $2 \mathbf{f}$ benzofulvene $\mathbf{5 f}$ was only obtained in a poor $8 \%$ yield (entries 5 and 6). Primary and secondary alkynols $\mathbf{2 g}$,h bearing an electron-rich 4-methoxyphenyl group instead of phenyl at the propargylic position also reacted to give the corresponding benzofulvenes $\mathbf{5 g}, \mathbf{h}$ although in moderate yields and selectivity (entries 7 and 8). For substrate $\mathbf{2 i}$, bearing an electron-withdrawing group (chlorine) on the benzene ring and a methyl group as $\mathrm{R}^{2}$ substituent, the reaction proceeded smoothly to afford the $(E)$-isomer of benzofulvene $\mathbf{5 i}$ in high yield (entry 9). However, changing the aliphatic substituent at the propargylic position adjacent to the indole $\left(\mathrm{R}^{1}\right)$ from cyclopropyl to other alkyl groups including methyl or $i$-propyl (substrates $\mathbf{2} \mathbf{j}-\mathbf{l}$ ) led to a decrease in the selectivity and so, the corresponding benzofulvenes $\mathbf{5 j}$-l could only be isolated in moderate yields (entries 10-12). The presence of a thiophene group at the propargylic position in $\mathbf{2 k}$ allowed the preparation of a 5-indolyl-4methylenecyclopenta $[b]$ thiophene derivative $\mathbf{5 k}$. This product resulted to be acid-sensitive and could only be isolated in low yield (entry 11). 

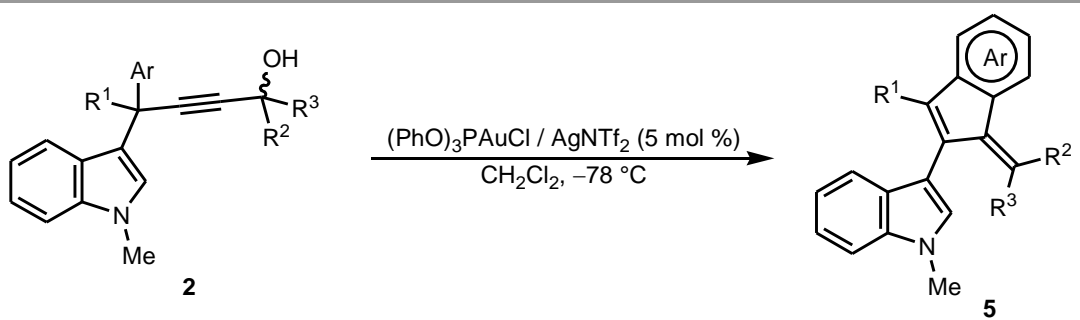

\begin{tabular}{|c|c|c|c|c|c|c|c|c|}
\hline Entry & Alkynol & Ar & $\mathrm{R}^{1}$ & $\mathrm{R}^{2}$ & $\mathrm{R}^{3}$ & Ratio 5 / $4^{\mathrm{a}}$ & Product & Yield $(\%)^{\mathrm{b}}$ \\
\hline 1 & $2 \mathbf{a}$ & $\mathrm{Ph}$ & $c-\mathrm{C}_{3} \mathrm{H}_{5}$ & $n-\operatorname{Pr}$ & $\mathrm{H}$ & $7 / 1$ & $5 \mathbf{a}$ & 75 \\
\hline 2 & $2 b$ & $\mathrm{Ph}$ & $c-\mathrm{C}_{3} \mathrm{H}_{5}$ & $\mathrm{H}$ & $\mathrm{H}$ & $9 / 1$ & $5 \mathbf{b}$ & 77 \\
\hline 3 & $2 c$ & $\mathrm{Ph}$ & $c-\mathrm{C}_{3} \mathrm{H}_{5}$ & $4-\mathrm{ClC}_{6} \mathrm{H}_{4}$ & $\mathrm{H}$ & $>10 / 1$ & $5 c$ & 80 \\
\hline 4 & $2 d$ & $\mathrm{Ph}$ & $c-\mathrm{C}_{3} \mathrm{H}_{5}$ & $\mathrm{Me}$ & $\mathrm{H}$ & $6 / 1$ & $5 d$ & 84 \\
\hline 5 & $2 \mathrm{e}$ & $\mathrm{Ph}$ & $c-\mathrm{C}_{3} \mathrm{H}_{5}$ & $\mathrm{Me}$ & $\mathrm{Me}$ & $1 / 1.5$ & $5 e$ & $32^{c}$ \\
\hline 6 & $2 f$ & $\mathrm{Ph}$ & $c-\mathrm{C}_{3} \mathrm{H}_{5}$ & 4- $\mathrm{ClC}_{6} \mathrm{H}_{4}$ & 4- $\mathrm{ClC}_{6} \mathrm{H}_{4}$ & $1 / 7$ & 5f & $8^{\mathrm{d}}$ \\
\hline 7 & $2 \mathrm{~g}$ & $4-\mathrm{MeOC}_{6} \mathrm{H}_{4}$ & $c-\mathrm{C}_{3} \mathrm{H}_{5}$ & $\mathrm{H}$ & $\mathrm{H}$ & $2 / 1$ & $5 g$ & 45 \\
\hline 8 & $2 \mathbf{h}$ & 4- $\mathrm{MeOC}_{6} \mathrm{H}_{4}$ & $c-\mathrm{C}_{3} \mathrm{H}_{5}$ & $4-\mathrm{ClC}_{6} \mathrm{H}_{4}$ & $\mathrm{H}$ & $3 / 1$ & $5 \mathbf{h}$ & 51 \\
\hline 9 & $2 \mathbf{i}$ & 4- $\mathrm{ClC}_{6} \mathrm{H}_{4}$ & $c-\mathrm{C}_{3} \mathrm{H}_{5}$ & $\mathrm{Me}$ & $\mathrm{H}$ & $7 / 1$ & $5 \mathbf{i}$ & 78 \\
\hline 10 & $2 \mathbf{j}$ & $\mathrm{Ph}$ & $\mathrm{Me}$ & $\mathrm{H}$ & $\mathrm{H}$ & $3 / 1$ & $5 \mathbf{j}$ & $35^{\mathrm{e}}$ \\
\hline \multirow[t]{2}{*}{11} & $2 \mathbf{k}$ & 2-Thienyl & $\mathrm{Me}$ & $\mathrm{H}$ & $\mathrm{H}$ & $-{ }^{\mathrm{f}}$ & $5 \mathbf{k}$ & $30^{\mathrm{g}}$ \\
\hline & 21 & $\mathrm{Ph}$ & $i$-Pr & $\mathrm{Me}$ & $\mathrm{H}$ & $1.5 / 1$ & $5 \mathbf{l}^{\mathrm{h}}$ & $50^{\mathrm{i}}$ \\
\hline
\end{tabular}

${ }^{\mathrm{a}}$ Determined by ${ }^{1} \mathrm{H}$ NMR analysis of the crude reaction mixture.

${ }^{\mathrm{b}}$ Isolated yield after column chromatography.

c $52 \%$ of 4 e was also isolated.

$\mathrm{d} 70 \%$ of $\mathbf{4 f}$ was also isolated.

e $18 \%$ of $\mathbf{4} \mathbf{j}$ was also isolated.

${ }^{\mathrm{f}}$ Not determined.

${ }^{\mathrm{g}}$ Partial decomposition of the product under purification on silica gel chromatography.

${ }^{\text {h }}$ Obtained as a ca. $5 / 1$ mixture of $E / Z$ isomers.

i $31 \%$ of $\mathbf{4 l}$ was also isolated.

A possible mechanism for this tandem process is depicted in Scheme 4. Accordingly with our previous theoretical and experimental studies, ${ }^{15}$ initial coordination of the gold complex to the triple bond of the starting alkynol $\mathbf{2}$ would trigger an intramolecular attack of the indole on the activated alkyne affording an alkylidenecyclopropane intermediate $\mathbf{1 0}$. Subsequent rearrangement and 1,2-migration of the indole nucleus through a torquoselective $4 \pi \mathrm{e}^{-}$electrocyclic ring opening gives rise to a gold carbenoid / carbocation species 11. Then, a gold variant of the iso-Nazarov cyclization takes place on the gold-stabilized carbocation leading to intermediate 12. At this point usual protodemetalation would render the expected alcohols $\mathbf{4}$ regenerating the catalytic gold species. To account for the generation of benzofulvenes $\mathbf{5}$, we tentatively propose that some type of interaction between gold and oxygen atoms could induce a competitive elimination process that finally leads to the loss of water, in a concerted or a stepwise manner, with the subsequent recovery of the cationic gold complex and release of the final methyleneindene derivative 5 (Scheme 4). From the obtained experimental results, the preferred pathway seems to be dependent on the nature of the ligand in the gold complex, the temperature of the reaction, the nature of the alkyl group $\left(\mathrm{R}^{1}\right)$ at the propargylic position, and mainly on the steric hindrance of the hydroxyl group. So, with primary or secondary alcohols the coordination of the hydroxyl group with the metallic center could be favoured compared with more encumbered tertiary alcohols leading to a higher selectivity for the elimination pathway. These facts also support that the generation of benzofulvenes $\mathbf{5}$ is not due to an elimination of water from alcohols $\mathbf{4}$ that would be more favored with highly substituted alcohols.

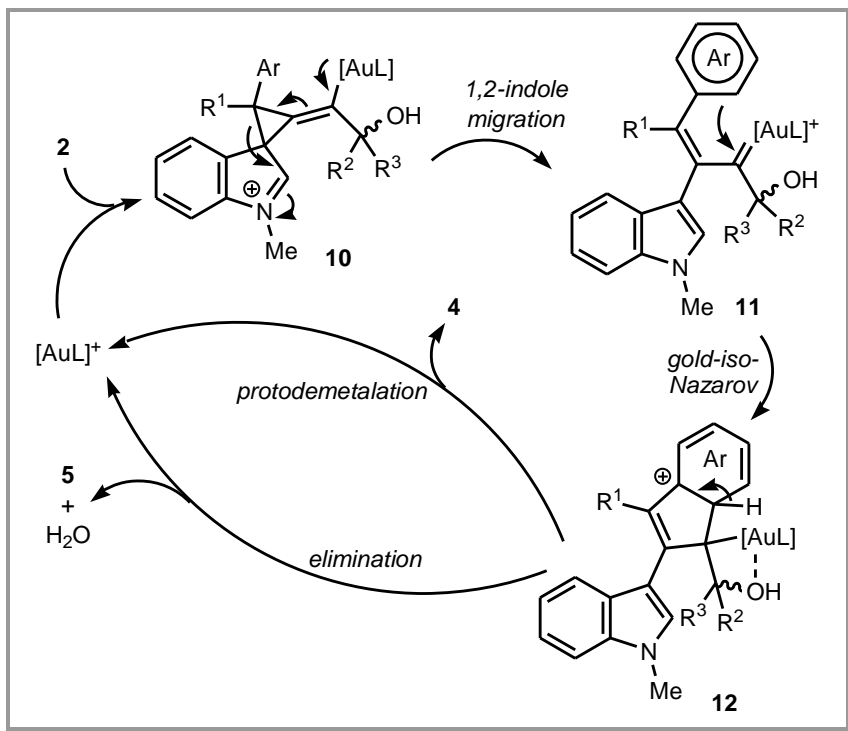

Scheme 4 Proposed Mechanism for the Formation of Benzofulvenes 5

In summary, we have developed a gold-catalyzed intramolecular transformation of 3-propargylindoles to give new and interesting benzofulvene derivatives with an indol-3-yl substituent at the C-2 position in 
moderate to good yields under mild conditions. This process involves a tandem 1,2-indole migration / auraiso-Nazarov cyclization / elimination sequence.

All reactions involving air-sensitive compounds were carried out under a $\mathrm{N}_{2}$ atmosphere, in oven-dried glassware, with magnetic stirring. Temperatures are reported as bath temperatures. Solvents used in extraction and purification were distilled prior to use. TLC was performed on alumina-backed plates coated with silica gel 60 with F254 indicator and the chromatograms were visualized by UV light $(254 \mathrm{~nm}$ and/or $366 \mathrm{~nm}$ ) and/or by staining with a Ce/Mo reagent and subsequent heating. $R_{f}$ values refer to silica gel. NMR spectra were measured on Varian Mercury-Plus $300 \mathrm{MHz}$ and Varian Inova- $400 \mathrm{MHz}$ spectrometers. ${ }^{1} \mathrm{H}$ NMR: splitting pattern abbreviations are: s, singlet; bs, broad singlet; d, doublet; t, triplet; q, quartet; quint, quintet; sept, septet; $\mathrm{m}$, multiplet; the chemical shifts are reported in ppm using residual solvent peak as reference. ${ }^{13} \mathrm{C}$ NMR spectra were recorded at $75.4 \mathrm{MHz}$ or 100.6 $\mathrm{MHz}$ using broadband proton decoupling and chemical shifts are reported in ppm using residual solvent peaks as reference $\left(\mathrm{CDCl}_{3}: \delta 77.16\right)$ and the multiplicities were determined by DEPT experiments. High resolution mass spectra (HRMS) were recorded on a Micromass Autospec spectrometer using EI at $70 \mathrm{eV}$. Melting points were measured on a Gallenkamp apparatous using open capillary tubes and are uncorrected. GC-MS and low resolution mass spectra (LRMS) measurements were recorded on an Agilent 6890N/5973 Network GC System, equipped with a HP-5MS column.

\section{Synthesis of Alkynols 2a-c,e-h; 1-cyclopropyl-1-(1- methyl-1 $H$-indol-3-yl)-1-phenylhept-2-yn-4-ol (2a); Typical Procedure}

BuLi $(1.31 \mathrm{~mL}$ of $1.6 \mathrm{M}$ solution in hexane, 2.1 mmol) was added to a solution of the corresponding 3-propargylindole $8(571 \mathrm{mg}, 2 \mathrm{mmol})$ in dry THF (2 $\mathrm{mL}$ ) at $-78^{\circ} \mathrm{C}$. The mixture was stirred from $-78{ }^{\circ} \mathrm{C}$ to r.t. for $1 \mathrm{~h}$ and then recooled to $-78{ }^{\circ} \mathrm{C}$. Butyraldehyde (159 mg, $2.2 \mathrm{mmol}$ ) was slowly added and the resulting mixture was stirred at r.t. for $2 \mathrm{~h} .10$ $\mathrm{mL}$ of saturated $\mathrm{NH}_{4} \mathrm{Cl}$ were added and the separated aqueous phase was extracted with $\mathrm{Et}_{2} \mathrm{O}(2 \times 20 \mathrm{~mL})$ and the combined organic layers were dried over anhydrous $\mathrm{Na}_{2} \mathrm{SO}_{4}$. The solvent was removed under reduced pressure and the crude residue was purified by flash column chromatography on silica gel using using hexane-EtOAc (4:1) as eluent to afford 2a as a 1:1 mixture of diastereoisomers; pale yellow foam; yield: $628 \mathrm{mg}$ (88\%); $R_{f}=0.15$ (hexane-EtOAc, 4:1).

${ }^{1} \mathrm{H}$ NMR $\left(300 \mathrm{MHz}, \mathrm{CDCl}_{3}\right): \delta=0.47-0.74(\mathrm{~m}, 4 \mathrm{H}$, $\left.\mathrm{CH}_{2} \mathrm{CH}_{2} \mathrm{CH}\right), \quad 0.89-0.96 \quad\left(\mathrm{~m}, 3 \mathrm{H}, \quad \mathrm{CH}_{2} \mathrm{CH}_{2} \mathrm{CH}_{3}\right)$,
1.39-1.74 (m, 6H, $\left.\mathrm{CH}_{2} \mathrm{CH}_{2} \mathrm{CH}+\mathrm{CH}_{2} \mathrm{CH}_{2} \mathrm{CH}_{3}+\mathrm{OH}\right)$, $3.81\left(\mathrm{~s}, 3 \mathrm{H}, \mathrm{NCH}_{3}\right), 4.41(\mathrm{t}, J=6.6 \mathrm{~Hz}, 1 \mathrm{H}, \mathrm{CHOH})$, 6.86-6.93 (m, 1H, ArH), 7.11-7.30 (m, 7H, ArH), $7.47-7.53(\mathrm{~m}, 2 \mathrm{H}, \mathrm{ArH})$. The signals of both isomers appear completely overlapped.

${ }^{13} \mathrm{C}$ NMR $\left(75.4 \mathrm{MHz}, \mathrm{CDCl}_{3}\right): \delta=2.5\left(\mathrm{CH}_{2}\right), 2.6$ $\left(\mathrm{CH}_{2}\right), \quad 3.8\left(\mathrm{CH}_{2}\right), \quad 3.9\left(\mathrm{CH}_{2}\right), 13.9\left(\mathrm{CH}_{3}\right.$, both isomers), $18.6\left(\mathrm{CH}_{2}\right.$, both isomers $), 21.3(\mathrm{CH}), 21.4$ $(\mathrm{CH}), \quad 32.9\left(\mathrm{CH}_{3}\right.$, both isomers $), 40.3\left(\mathrm{CH}_{2}\right.$, both isomers), 45.5 (C, both isomers), $62.6(\mathrm{CH}$, both isomers), 85.5 (C, both isomers), $85.76(\mathrm{C}), 85.78(\mathrm{C})$, $109.3(\mathrm{CH}$, both isomers), $118.80(\mathrm{CH}), 118.81(\mathrm{CH})$, $119.7(\mathrm{C}), 119.8(\mathrm{C}), 121.2$ (CH, both isomers), 121.6 (CH, both isomers), 126.2 (C, both isomers), 126.5 $(\mathrm{CH}$, both isomers), $127.10(\mathrm{CH}), 127.12(\mathrm{CH}), 127.2$ ( $2 \times \mathrm{CH}$, both isomers), $128.1(2 \times \mathrm{CH}$, both isomers), 137.8 (C, both isomers), 145.68 (C), 145.71 (C).

MS (EI, $70 \mathrm{eV}): m / z(\%)=357\left(64, \mathrm{M}^{+}\right), 329(100)$, 157 (68), 144 (95).

HRMS-EI: $m / z$ [M] $]^{+}$calcd for $\mathrm{C}_{25} \mathrm{H}_{27} \mathrm{NO}: 357.2093$; found: 357.2086.

2b, white solid; yield: $479 \mathrm{mg}(76 \%) ; R_{f}=0.19$ (hexane- $\mathrm{Et}_{2} \mathrm{O}, 1: 1$ ); $\mathrm{mp}=54-56{ }^{\circ} \mathrm{C}$.

${ }^{1} \mathrm{H}$ NMR $\left(300 \mathrm{MHz}, \mathrm{CDCl}_{3}\right): \delta=0.55-0.76(\mathrm{~m}, 4 \mathrm{H}$, $\mathrm{CH}_{2} \mathrm{CH}_{2} \mathrm{CH}$ ), 1.55 (bs, $\left.1 \mathrm{H}, \mathrm{OH}\right), 1.56-1.67(\mathrm{~m}, 1 \mathrm{H}$, $\left.\mathrm{CH}_{2} \mathrm{CH}_{2} \mathrm{CH}\right), 3.81\left(\mathrm{~s}, 3 \mathrm{H}, \mathrm{NCH}_{3}\right), 4.29(\mathrm{~s}, 2 \mathrm{H}$, $\left.\mathrm{CH}_{2} \mathrm{OH}\right), 6.87-6.94(\mathrm{~m}, 1 \mathrm{H}, \mathrm{ArH}), 7.12-7.31(\mathrm{~m}, 7 \mathrm{H}$, $\mathrm{ArH}), 7.50-7.56$ (m, 2H, ArH).

${ }^{13} \mathrm{C}$ NMR $\left(75.4 \mathrm{MHz}, \mathrm{CDCl}_{3}\right): \delta=2.5\left(\mathrm{CH}_{2}\right), 3.8$ $\left(\mathrm{CH}_{2}\right), 21.3(\mathrm{CH}), 32.8\left(\mathrm{CH}_{3}\right), 45.5(\mathrm{C}), 51.3\left(\mathrm{CH}_{2}\right)$, $82.6(\mathrm{C}), 86.4(\mathrm{C}), 109.3(\mathrm{CH}), 118.8(\mathrm{CH}), 119.5(\mathrm{C})$, $121.1(\mathrm{CH}), 121.6(\mathrm{CH}), 126.0(\mathrm{C}), 126.6(\mathrm{CH})$, 127.20 $(\mathrm{CH}), 127.22(2 \times \mathrm{CH}), 128.1(2 \times \mathrm{CH}), 137.8$ (C), 145.4 (C).

MS (EI, $70 \mathrm{eV}): m / z(\%)=315\left(38, \mathrm{M}^{+}\right), 287(49)$, 274 (33), 157 (54), 144 (100), 131 (32).

HRMS-EI: $m / z[\mathrm{M}]^{+}$calcd for $\mathrm{C}_{22} \mathrm{H}_{21} \mathrm{NO}$ : 315.1623 ; found: 315.1628 .

2c isolated as a $\sim 1: 1$ mixture of diastereoisomers; white foam; yield: $663 \mathrm{mg}(78 \%) ; R_{f}=0.15$ (hexane-EtOAc, 4:1).

${ }^{1} \mathrm{H}$ NMR $\left(300 \mathrm{MHz}, \mathrm{CDCl}_{3}\right): \delta=0.56-0.78(\mathrm{~m}, 4 \mathrm{H}$, $\left.\mathrm{CH}_{2} \mathrm{CH}_{2} \mathrm{CH}\right), 1.62-1.71\left(\mathrm{~m}, 1 \mathrm{H}, \mathrm{CH}_{2} \mathrm{CH}_{2} \mathrm{CH}\right), 2.46$ (bs, $1 \mathrm{H}, \mathrm{OH}), 3.80\left(\mathrm{~s}, 3 \mathrm{H}, \mathrm{NCH}_{3}\right), 5.43(\mathrm{~s}, 1 \mathrm{H}$, $\mathrm{CHOH}), 6.90-6.97(\mathrm{~m}, 1 \mathrm{H}, \mathrm{ArH}), 7.17-7.36(\mathrm{~m}, 9 \mathrm{H}$, ArH), $7.41(\mathrm{~d}, J=8.3 \mathrm{~Hz}, 2 \mathrm{H}, \mathrm{ArH}), 7.53-7.58(\mathrm{~m}$, $2 \mathrm{H}, \mathrm{ArH})$. The signals of both isomers appear completely overlapped.

${ }^{13} \mathrm{C}$ NMR $\left(75.4 \mathrm{MHz}, \mathrm{CDCl}_{3}\right): \delta=2.59\left(\mathrm{CH}_{2}\right), 2.62$ $\left(\mathrm{CH}_{2}\right), 3.9\left(\mathrm{CH}_{2}\right.$, both isomers $), 21.5(\mathrm{CH}$, both isomers), $32.9\left(\mathrm{CH}_{3}\right.$, both isomers $), 45.7(\mathrm{C}$, both isomers), $64.1(\mathrm{CH}$, both isomers), $83.9(\mathrm{C}), 84.0(\mathrm{C})$, $88.26(\mathrm{C}), 88.29(\mathrm{C}), 109.3(\mathrm{CH}$, both isomers), 118.9 $(\mathrm{CH}$, both isomers), 119.35 (C), $119.39(\mathrm{C}), 121.2$ 
( $\mathrm{CH}$, both isomers), $121.7(\mathrm{CH}$, both isomers), 126.1 (C, both isomers), 126.7 ( $\mathrm{CH}$, both isomers), 127.2 (3 $\times \mathrm{CH}$, both isomers), $128.1(2 \times \mathrm{CH}$, both isomers $)$, $128.2(2 \times \mathrm{CH}$, both isomers $), 128.6(2 \times \mathrm{CH}$, both isomers), 133.91 (C), 133.94 (C), 137.8 (C, both isomers), 139.6 (C, both isomers), 145.20 (C), 145.23 (C).

MS (EI, $70 \mathrm{eV}): m / z(\%)=425\left(42, \mathrm{M}^{+}\right), 397(52)$, 284 (59), 157 (65).

HRMS-EI: $m / z \quad[\mathrm{M}]^{+}$calcd for $\mathrm{C}_{28} \mathrm{H}_{24} \mathrm{ClNO}$ : 425.1546; found: 425.1546 .

2e, white solid; yield: $466 \mathrm{mg}(68 \%) ; R_{f}=0.20$ (hexane-EtOAc, 3:1); $\mathrm{mp}=55-57{ }^{\circ} \mathrm{C}$.

${ }^{1} \mathrm{H}$ NMR $\left(300 \mathrm{MHz}, \mathrm{CDCl}_{3}\right): \delta=0.54-0.86(\mathrm{~m}, 4 \mathrm{H}$, $\left.\mathrm{CH}_{2} \mathrm{CH}_{2} \mathrm{CH}\right), 1.57\left(\mathrm{~s}, 6 \mathrm{H}, \mathrm{CH}_{3} \mathrm{CCH}_{3}\right), 1.60-1.70(\mathrm{~m}$, $1 \mathrm{H}, \mathrm{CH}_{2} \mathrm{CH}_{2} \mathrm{CH}$ ), 2.19 (bs, $\left.1 \mathrm{H}, \mathrm{OH}\right), 3.81$ (s, $3 \mathrm{H}$, $\left.\mathrm{NCH}_{3}\right), 6.93-7.01(\mathrm{~m}, 1 \mathrm{H}, \mathrm{ArH}), 7.18-7.37(\mathrm{~m}, 7 \mathrm{H}$, $\mathrm{ArH}), 7.56-7.62$ (m, 2H, ArH).

${ }^{13} \mathrm{C}$ NMR $\left(75.4 \mathrm{MHz}, \mathrm{CDCl}_{3}\right): \delta=2.5\left(\mathrm{CH}_{2}\right), 3.8$ $\left(\mathrm{CH}_{2}\right), 21.3(\mathrm{CH}), 31.67\left(\mathrm{CH}_{3}\right), 31.71\left(\mathrm{CH}_{3}\right), 32.8$ $\left(\mathrm{CH}_{3}\right), 45.3(\mathrm{C}), 65.4(\mathrm{C}), 82.8(\mathrm{C}), 89.4(\mathrm{C}), 109.2$ $(\mathrm{CH}), 118.7(\mathrm{CH}), 119.8(\mathrm{C}), 121.2(\mathrm{CH}), 121.5(\mathrm{CH})$, $126.3(\mathrm{C}), 126.5(\mathrm{CH}), 127.0(\mathrm{CH}), 127.1(2 \times \mathrm{CH})$, $128.0(2 \times \mathrm{CH}), 137.8(\mathrm{C}), 145.9(\mathrm{C})$.

MS (EI, $70 \mathrm{eV}): m / z(\%)=343\left(54, \mathrm{M}^{+}\right), 315(100)$, 302 (41), 157 (41), 144 (61).

HRMS-EI: $m / z[\mathrm{M}]^{+}$calcd for $\mathrm{C}_{24} \mathrm{H}_{25} \mathrm{NO}: 343.1936$; found: 343.1939 .

2f, white solid; yield: $644 \mathrm{mg}(60 \%) ; R_{f}=0.22$ (hexane- $\mathrm{Et}_{2} \mathrm{O}, 3: 1$ ); $\mathrm{mp}=80-82^{\circ} \mathrm{C}$.

${ }^{1} \mathrm{H}$ NMR $\left(300 \mathrm{MHz}, \mathrm{CDCl}_{3}\right): \delta=0.51-0.71(\mathrm{~m}, 4 \mathrm{H}$, $\left.\mathrm{CH}_{2} \mathrm{CH}_{2} \mathrm{CH}\right), 1.58-1.69\left(\mathrm{~m}, 1 \mathrm{H}, \mathrm{CH}_{2} \mathrm{CH}_{2} \mathrm{CH}\right), 2.73$ (bs, $1 \mathrm{H}, \mathrm{OH}), 3.81\left(\mathrm{~s}, 3 \mathrm{H}, \mathrm{NCH}_{3}\right), 6.85-6.92(\mathrm{~m}, 1 \mathrm{H}$, ArH), 7.15-7.33 (m, 11H, ArH), 7.37-7.51 (m, 6H, ArH).

${ }^{13} \mathrm{C}$ NMR $\left(75.4 \mathrm{MHz}, \mathrm{CDCl}_{3}\right): \delta=2.7\left(\mathrm{CH}_{2}\right), 4.1$ $\left(\mathrm{CH}_{2}\right), 21.6(\mathrm{CH}), 33.0\left(\mathrm{CH}_{3}\right), 45.9(\mathrm{C}), 73.9(\mathrm{C}), 86.4$ (C), $89.9(\mathrm{C}), 109.4(\mathrm{CH}), 119.0(\mathrm{CH}), 119.3(\mathrm{C})$, $121.2(\mathrm{CH}), 121.8(\mathrm{CH}), 126.2(\mathrm{C}), 126.8(\mathrm{CH})$, $127.18(2 \times \mathrm{CH}), 127.23(\mathrm{CH}), 127.6(4 \times \mathrm{CH}), 128.2$ $(2 \times \mathrm{CH}), 128.45(2 \times \mathrm{CH}), 128.46(2 \times \mathrm{CH}), 133.7(2$ $\times \mathrm{C}), 137.9(\mathrm{C}), 143.6(2 \times \mathrm{C}), 145.2(\mathrm{C})$.

2g, white solid; yield: $442 \mathrm{mg}(64 \%) ; R_{f}=0.19$ (hexane- $\mathrm{Et}_{2} \mathrm{O}, 1: 2$ ); $\mathrm{mp}=62-64{ }^{\circ} \mathrm{C}$.

${ }^{1} \mathrm{H}$ NMR $\left(300 \mathrm{MHz}, \mathrm{CDCl}_{3}\right): \delta=0.52-0.74(\mathrm{~m}, 4 \mathrm{H}$, $\left.\mathrm{CH}_{2} \mathrm{CH}_{2} \mathrm{CH}\right), 1.56-1.64\left(\mathrm{~m}, 2 \mathrm{H}, \mathrm{CH}_{2} \mathrm{CH}_{2} \mathrm{CH}+\mathrm{OH}\right)$, $3.78\left(\mathrm{~s}, 3 \mathrm{H}, \mathrm{NCH}_{3}\right.$ or $\left.\mathrm{OCH}_{3}\right), 3.80\left(\mathrm{~s}, 3 \mathrm{H}, \mathrm{NCH}_{3}\right.$ or $\left.\mathrm{OCH}_{3}\right), 4.28\left(\mathrm{~s}, 2 \mathrm{H}, \mathrm{CH}_{2} \mathrm{OH}\right), 6.81(\mathrm{~d}, J=8.9 \mathrm{~Hz}, 2 \mathrm{H}$, ArH), 6.88-6.95 (m, 1H, ArH), 7.13-7.31 (m, 4H, $\mathrm{ArH}), 7.43$ (d, $J=8.9 \mathrm{~Hz}, 2 \mathrm{H}, \mathrm{ArH})$.

${ }^{13} \mathrm{C}$ NMR $\left(75.4 \mathrm{MHz}, \mathrm{CDCl}_{3}\right): \delta=2.6\left(\mathrm{CH}_{2}\right), 3.7$ $\left(\mathrm{CH}_{2}\right), 21.4(\mathrm{CH}), 32.9\left(\mathrm{CH}_{3}\right), 44.9(\mathrm{C}), 51.6\left(\mathrm{CH}_{2}\right)$,
$55.3\left(\mathrm{CH}_{3}\right), 82.4(\mathrm{C}), 87.0(\mathrm{C}), 109.3(\mathrm{CH}), 113.4(2 \times$ $\mathrm{CH}), 118.9(\mathrm{CH}), 119.8(\mathrm{C}), 121.3(\mathrm{CH}), 121.6(\mathrm{CH})$, $126.1(\mathrm{C}), 127.2(\mathrm{CH}), 128.4(2 \times \mathrm{CH}), 137.6(\mathrm{C})$, 137.8 (C), $158.2(\mathrm{C})$.

MS (EI, $70 \mathrm{eV}): m / z(\%)=345\left(67, \mathrm{M}^{+}\right), 317(100)$, 304 (88), 157 (46), 144 (43).

HRMS-EI: $m / z[\mathrm{M}]^{+}$calcd for $\mathrm{C}_{23} \mathrm{H}_{23} \mathrm{NO}_{2}: 345.1729$; found: 345.1726 .

2h isolated as a $\sim 1: 1$ mixture of diastereoisomers; brown solid; yield: $601 \mathrm{mg}(66 \%) ; R_{f}=0.19$ (hexane- $\mathrm{Et}_{2} \mathrm{O}, 2: 1$ ); $\mathrm{mp}=55-57{ }^{\circ} \mathrm{C}$.

${ }^{1} \mathrm{H}$ NMR $\left(300 \mathrm{MHz}, \mathrm{CDCl}_{3}\right): \delta=0.54-0.92(\mathrm{~m}, 4 \mathrm{H}$, $\left.\mathrm{CH}_{2} \mathrm{CH}_{2} \mathrm{CH}\right), 1.57-1.69\left(\mathrm{~m}, 1 \mathrm{H}, \mathrm{CH}_{2} \mathrm{CH}_{2} \mathrm{CH}\right), 3.77$ (s, $3 \mathrm{H}, \mathrm{NCH}_{3}$ or $\left.\mathrm{OCH}_{3}\right), 3.79\left(\mathrm{~s}, 3 \mathrm{H}, \mathrm{NCH}_{3}\right.$ or $\left.\mathrm{OCH}_{3}\right)$, 4.57 (bs, $1 \mathrm{H}, \mathrm{OH}), 5.43(\mathrm{~d}, J=4.9 \mathrm{~Hz}, 1 \mathrm{H}, \mathrm{CHOH})$, 6.73-6.83 (m, 2H, ArH), 6.88-6.95 (m, 1H, ArH), 7.05-7.10 (m, 1H, ArH), 7.13-7.49 (m, 9H, ArH). The signals of both isomers appear completely overlapped.

${ }^{13} \mathrm{C}$ NMR $\left(75.4 \mathrm{MHz}, \mathrm{CDCl}_{3}\right): \delta=2.6\left(\mathrm{CH}_{2}\right.$, both isomers $), 3.8\left(\mathrm{CH}_{2}\right.$, both isomers $), 21.6(\mathrm{CH}$, both isomers), $32.9\left(\mathrm{CH}_{3}\right.$, both isomers $), 45.0(\mathrm{C}$, both isomers $), 55.3\left(\mathrm{CH}_{3}\right.$, both isomers $), 64.1(\mathrm{CH}$, both isomers), $83.65(\mathrm{C}), 83.67(\mathrm{C}), 88.6$ (C), 88.7 (C), $109.4(\mathrm{CH}$, both isomers), $113.4(2 \times \mathrm{CH}$, both isomers), $118.9(\mathrm{CH}$, both isomers), $119.6(\mathrm{C}$, both isomers), $121.3(\mathrm{CH}$, both isomers), $121.7(\mathrm{CH}$, both isomers), 126.1 (C, both isomers), $127.1(\mathrm{CH}$, both isomers $), 128.16(2 \times \mathrm{CH}), 128.20(2 \times \mathrm{CH}), 128.3(2$ $\times \mathrm{CH}$, both isomers), $128.65(2 \times \mathrm{CH}), 128.66(2 \times$ $\mathrm{CH}), 133.9$ (C), 134.0 (C), 137.3 (C), 137.4 (C), 137.8 (C, both isomers), 139.6 (C, both isomers), 158.2 (C, both isomers).

MS (EI, $70 \mathrm{eV}): \mathrm{m} / \mathrm{z}(\%)=455\left(39, \mathrm{M}^{+}\right), 427(70)$, 314 (100), 157 (53).

HRMS-EI: $m / z \quad[\mathrm{M}]^{+}$calcd for $\mathrm{C}_{29} \mathrm{H}_{26} \mathrm{ClNO}_{2}$ : 455.1652; found: 455.1651 .

\section{Synthesis of 1-cyclopropyl-1-(1-methyl-1H-indol-3- yl)-1-phenylhept-2-yn-4-yl acetate (1a)}

To a solution of alkynol $\mathbf{2 a}(179 \mathrm{mg}, 0.5 \mathrm{mmol})$ in $\mathrm{CH}_{2} \mathrm{Cl}_{2}(1 \mathrm{~mL})$ were added successively triethylamine (202 mg, $2 \mathrm{mmol}$ ), 4-dimethylaminopyridine (12 mg, $0.1 \mathrm{mmol})$ and acetic anhydride $(102 \mathrm{mg}, 1 \mathrm{mmol})$. The reaction was stirred overnight, cooled to $0^{\circ} \mathrm{C}$ and quenched with saturated $\mathrm{NH}_{4} \mathrm{Cl}$. The resulting mixture was extracted with $\mathrm{Et}_{2} \mathrm{O}(2 \times 10 \mathrm{~mL})$ and the combined organic layers were washed with brine (10 $\mathrm{mL}$ ) and dried over anhydrous $\mathrm{Na}_{2} \mathrm{SO}_{4}$. The solvent was removed under reduced pressure and the crude residue was purified by flash column chromatography on silica gel using hexane- $\mathrm{Et}_{2} \mathrm{O}(3.5: 1)$ as eluent to afford 1a as a $\sim 1: 1$ mixture of diastereoisomers; yellow oil; yield: $156 \mathrm{mg}(78 \%) ; \quad R_{f}=0.22$ (hexane- $\mathrm{Et}_{2} \mathrm{O}, 3.5: 1$ ). 
${ }^{1} \mathrm{H}$ NMR $\left(300 \mathrm{MHz}, \mathrm{CDCl}_{3}\right): \delta=0.49-0.74(\mathrm{~m}, 4 \mathrm{H}$, $\left.\mathrm{CH}_{2} \mathrm{CH}_{2} \mathrm{CH}\right), 0.88-0.92\left(\mathrm{~m}, 3 \mathrm{H}, \quad \mathrm{CH}_{2} \mathrm{CH}_{2} \mathrm{CH}_{3}\right)$, 1.39-1.46 (m, 2H, $\left.\mathrm{CH}_{2} \mathrm{CH}_{2} \mathrm{CH}_{3}\right), 1.55-1.76(\mathrm{~m}, 3 \mathrm{H}$, $\left.\mathrm{CH}_{2} \mathrm{CH}_{2} \mathrm{CH}+\mathrm{CH}_{2} \mathrm{CH}_{2} \mathrm{CH}_{3}\right), 2.04$ (s, $\left.3 \mathrm{H}, \mathrm{OCOCH}_{3}\right)$, $3.80\left(\mathrm{~s}, 3 \mathrm{H}, \mathrm{NCH}_{3}\right), 5.38-5.44(\mathrm{~m}, 1 \mathrm{H}, \mathrm{CHOAc})$, 6.87-6.92 (m, 1H, ArH), 7.12-7.29 (m, 7H, ArH), 7.49-7.53 (m, 2H, ArH). The signals of both isomers are overlapped.

${ }^{13} \mathrm{C} \mathrm{NMR}\left(75.4 \mathrm{MHz}, \mathrm{CDCl}_{3}\right): \delta=2.50\left(\mathrm{CH}_{2}\right), 2.53$ $\left(\mathrm{CH}_{2}\right), 3.78\left(\mathrm{CH}_{2}\right), 3.83\left(\mathrm{CH}_{2}\right), 13.7\left(\mathrm{CH}_{3}\right.$, both isomers), $18.5\left(\mathrm{CH}_{2}\right.$, both isomers $), 21.1(\mathrm{CH}$, both isomers), $21.3\left(\mathrm{CH}_{3}\right.$, both isomers $), 32.8\left(\mathrm{CH}_{3}\right.$, both isomers), $37.0\left(\mathrm{CH}_{2}\right.$, both isomers), $45.5(\mathrm{C}$, both isomers), $64.38(\mathrm{CH}), 64.43(\mathrm{CH}), 82.1(\mathrm{C}), 82.2(\mathrm{C})$, $86.0(\mathrm{C}), 86.1(\mathrm{C}), 109.2(\mathrm{CH}$, both isomers $), 118.7$ $(\mathrm{CH}$, both isomers), $119.4(\mathrm{C}), 119.5(\mathrm{C}), 121.2(\mathrm{CH})$, $121.3(\mathrm{CH}), 121.5(\mathrm{CH}$, both isomers), $126.1(\mathrm{C}$, both isomers), $126.5(\mathrm{CH}$, both isomers), $127.07(\mathrm{CH})$, 127.09 $(\mathrm{CH}), 127.1(2 \times \mathrm{CH}), 127.2(2 \times \mathrm{CH}), 128.0$ $(2 \times \mathrm{CH}$, both isomers), 137.7 (C, both isomers), 145.58 (C), 145.60 (C), 170.1 (C), 170.2 (C).

MS (EI, $70 \mathrm{eV}): \mathrm{m} / z(\%)=399\left(58, \mathrm{M}^{+}\right), 371(53)$, 157 (55), 144 (100).

HRMS-EI: $m / z$ [M] ${ }^{+}$calcd for $\mathrm{C}_{27} \mathrm{H}_{29} \mathrm{NO}_{2}$ : 399.2198 ; found: 399.2196.

\section{Synthesis of Alkynols 2d,i-l; 5-cyclopropyl-5-(1- methyl-1H-indol-3-yl)-5-phenylpent-3-yn-2-ol (2d); Typical Procedure}

To a mixture of the corresponding alkynol $9(477 \mathrm{mg}$, $2.2 \mathrm{mmol}$ ) and $N$-methylindole $(262 \mathrm{mg}, 2 \mathrm{mmol})$ in MeCN (2 mL), PTSA (19 mg, $0.1 \mathrm{mmol}$ ) was added. The reaction was stirred at room temperature until the indole was consumed, as determined by GC-MS and/or TLC. Solvent was removed, $\mathrm{NaOH} 1 \mathrm{M}(10$ $\mathrm{mL})$ was added and the mixture extracted with $\mathrm{Et}_{2} \mathrm{O}(2$ $\times 10 \mathrm{~mL})$. The combined organic layers were dried over anhydrous $\mathrm{Na}_{2} \mathrm{SO}_{4}$ and the solvent was removed under reduced pressure. The residue was purified by silica gel column chromatography using hexane- $\mathrm{Et}_{2} \mathrm{O}$ (1.5:1) as eluent to afford $2 \mathbf{d}$ as a $\sim 1: 1$ mixture of diastereoisomers; white foam; yield: $474 \mathrm{mg}$ (72\%); $R_{f}=0.13$ (hexane- $\mathrm{Et}_{2} \mathrm{O}, 1.5: 1$ ).

${ }^{1} \mathrm{H}$ NMR $\left(300 \mathrm{MHz}, \mathrm{CDCl}_{3}\right): \delta=0.51-0.79(\mathrm{~m}, 4 \mathrm{H}$, $\left.\mathrm{CH}_{2} \mathrm{CH}_{2} \mathrm{CH}\right), 1.47\left(\mathrm{~d}, \mathrm{~J}=6.6 \mathrm{~Hz}, 3 \mathrm{H}, \mathrm{CHCH}_{3}\right)$, 1.56-1.67 (m, $\left.1 \mathrm{H}, \mathrm{CH}_{2} \mathrm{CH}_{2} \mathrm{CH}\right), 1.84$ (bs, $\left.1 \mathrm{H}, \mathrm{OH}\right)$, $3.81\left(\mathrm{~s}, 3 \mathrm{H}, \mathrm{NCH}_{3}\right), 4.55(\mathrm{q}, J=6.6 \mathrm{~Hz}, 1 \mathrm{H}, \mathrm{CHOH})$, 6.89-6.96 (m, 1H, ArH), 7.14-7.33 (m, 7H, ArH), 7.51-7.57 (m, 2H, ArH). The signals of both isomers appear completely overlapped.

${ }^{13} \mathrm{C}$ NMR $\left(75.4 \mathrm{MHz}, \mathrm{CDCl}_{3}\right): \delta=2.50\left(\mathrm{CH}_{2}\right), 2.54$ $\left(\mathrm{CH}_{2}\right), 3.80\left(\mathrm{CH}_{2}\right), 3.82\left(\mathrm{CH}_{2}\right), 21.30(\mathrm{CH}), 21.32$ $(\mathrm{CH}), 24.8\left(\mathrm{CH}_{3}\right.$, both isomers $), 32.9\left(\mathrm{CH}_{3}\right.$, both isomers), 45.4 (C, both isomers), $58.7(\mathrm{CH}$, both isomers), 84.8 (C, both isomers), 86.6 (C, both isomers), $109.3(\mathrm{CH}$, both isomers), $118.8(\mathrm{CH}), 118.9$ $(\mathrm{CH}), 119.7$ (C, both isomers), 121.2 (CH, both isomers), $121.6(\mathrm{CH}$, both isomers), 126.2 (C, both isomers), $126.6(\mathrm{CH}$, both isomers), $127.11(\mathrm{CH})$, $127.13(\mathrm{CH}), 127.2(2 \times \mathrm{CH}$, both isomers $), 128.1(2$ $\times \mathrm{CH}$, both isomers), 137.8 (C, both isomers), 145.6 (C), 145.7 (C).

MS (EI, $70 \mathrm{eV}): m / z(\%)=329\left(68, \mathrm{M}^{+}\right), 301(100)$, 288 (48), 157 (67), 144 (92).

HRMS-EI: $m / z[\mathrm{M}]^{+}$calcd for $\mathrm{C}_{23} \mathrm{H}_{23} \mathrm{NO}$ : 329.1780; found: 329.1778 .

$\mathbf{2 i}$, isolated as a $\sim 1: 1$ mixture of diastereoisomers; white solid; yield: $530 \mathrm{mg}(73 \%) ; \quad R_{f}=0.18$ (hexane-Et $\left.{ }_{2} \mathrm{O}, 1: 1\right) ; \mathrm{mp}=59-61{ }^{\circ} \mathrm{C}$.

${ }^{1} \mathrm{H}$ NMR $\left(300 \mathrm{MHz}, \mathrm{CDCl}_{3}\right): \delta=0.51-0.76(\mathrm{~m}, 4 \mathrm{H}$, $\left.\mathrm{CH}_{2} \mathrm{CH}_{2} \mathrm{CH}\right), 1.45\left(\mathrm{~d}, \mathrm{~J}=6.6 \mathrm{~Hz}, 3 \mathrm{H}, \mathrm{CHCH}_{3}\right)$, 1.51-1.62 (m, 1H, $\left.\mathrm{CH}_{2} \mathrm{CH}_{2} \mathrm{CH}\right), 1.83$ (bs, $\left.1 \mathrm{H}, \mathrm{OH}\right)$, $3.81\left(\mathrm{~s}, 3 \mathrm{H}, \mathrm{NCH}_{3}\right), 4.54(\mathrm{q}, J=6.6 \mathrm{~Hz}, 1 \mathrm{H}, \mathrm{CHOH})$, 6.90-6.98 (m, 1H, ArH), 7.15-7.32 (m, 6H, ArH), 7.42-7.49 ( $\mathrm{m}, 2 \mathrm{H}, \mathrm{ArH})$. The signals of both isomers appear completely overlapped.

${ }^{13} \mathrm{C}$ NMR (75.4 MHz, $\left.\mathrm{CDCl}_{3}\right): \delta=2.45\left(\mathrm{CH}_{2}\right), 2.49$ $\left(\mathrm{CH}_{2}\right), 3.84\left(\mathrm{CH}_{2}\right), 3.86\left(\mathrm{CH}_{2}\right), 21.25(\mathrm{CH}), 21.27$ $(\mathrm{CH}), \quad 24.7\left(\mathrm{CH}_{3}\right.$, both isomers $), 32.9\left(\mathrm{CH}_{3}\right.$, both isomers), 45.1 (C, both isomers), 58.6 $(\mathrm{CH}$, both isomers), 84.3 (C, both isomers), 86.9 (C, both isomers), $109.4(\mathrm{CH}$, both isomers), $118.97(\mathrm{CH})$, $118.99(\mathrm{CH}), 119.2(\mathrm{C}$, both isomers), $121.0(\mathrm{CH}$, both isomers), $121.8(\mathrm{CH}$, both isomers), $126.0(\mathrm{C}$, both isomers), $127.12(\mathrm{CH}), 127.13(\mathrm{CH}), 128.2(2 \times$ $\mathrm{CH}$, both isomers $), 128.7(2 \times \mathrm{CH}$, both isomers $)$, 132.3 (C, both isomers), 137.8 (C, both isomers), 144.32 (C), 144.34 (C).

MS (EI, $70 \mathrm{eV}): \mathrm{m} / \mathrm{z}(\%)=363\left(52, \mathrm{M}^{+}\right), 335(77)$, 322 (39), 157 (70), 144 (100).

HRMS-EI: $m / z \quad[\mathrm{M}]^{+}$calcd for $\mathrm{C}_{23} \mathrm{H}_{22} \mathrm{ClNO}$ : 363.1390; found: 363.1388 .

$2 \mathbf{j}$, brown foam; yield: $254 \mathrm{mg}(44 \%) ; R_{f}=0.19$ (hexane- $\mathrm{Et}_{2} \mathrm{O}, 1: 1$ ).

${ }^{1} \mathrm{H}$ NMR $\left(300 \mathrm{MHz}, \mathrm{CDCl}_{3}\right): \delta=2.02$ (bs, $\left.1 \mathrm{H}, \mathrm{OH}\right)$, $2.07\left(\mathrm{~s}, 3 \mathrm{H}, \mathrm{CCH}_{3}\right), 3.78\left(\mathrm{~s}, 3 \mathrm{H}, \mathrm{NCH}_{3}\right), 4.33(\mathrm{~s}, 2 \mathrm{H}$, $\left.\mathrm{CH}_{2} \mathrm{OH}\right), 6.97-7.07$ (m, 2H, ArH), 7.18-7.43 (m, 6H, $\mathrm{ArH}), 7.56-7.62(\mathrm{~m}, 2 \mathrm{H}, \mathrm{ArH})$.

${ }^{13} \mathrm{C}$ NMR $\left(75.4 \mathrm{MHz}, \mathrm{CDCl}_{3}\right): \delta=31.1\left(\mathrm{CH}_{3}\right), 32.8$ $\left(\mathrm{CH}_{3}\right), 39.4(\mathrm{C}), 51.5\left(\mathrm{CH}_{2}\right), 80.9(\mathrm{C}), 91.1(\mathrm{C}), 109.4$ $(\mathrm{CH}), 118.9(\mathrm{CH}), 119.9(\mathrm{C}), 121.1(\mathrm{CH}), 121.7(\mathrm{CH})$, $125.9(\mathrm{C}), 126.4(\mathrm{CH}), 126.5(\mathrm{CH}), 126.6(2 \times \mathrm{CH})$, $128.2(2 \times \mathrm{CH}), 137.8(\mathrm{C}), 145.9(\mathrm{C})$.

MS (EI, $70 \mathrm{eV}): \mathrm{m} / z(\%)=289\left(61, \mathrm{M}^{+}\right), 274(100)$, 258 (58), 83 (22).

HRMS-EI: $\mathrm{m} / z$ [M] ${ }^{+}$calcd for $\mathrm{C}_{20} \mathrm{H}_{19} \mathrm{NO}$ : 289.1467; found: 289.1478 .

2k, brown foam; yield: $401 \mathrm{mg}(68 \%) ; R_{f}=0.23$ (hexane- $\mathrm{Et}_{2} \mathrm{O}, 1: 1.5$ ). 
${ }^{1} \mathrm{H}$ NMR $\left(300 \mathrm{MHz}, \mathrm{CDCl}_{3}\right): \delta=1.65(\mathrm{bs}, 1 \mathrm{H}, \mathrm{OH})$, $2.13\left(\mathrm{~s}, 3 \mathrm{H}, \mathrm{CCH}_{3}\right), 3.75\left(\mathrm{~s}, 3 \mathrm{H}, \mathrm{NCH}_{3}\right), 4.34(\mathrm{~s}, 2 \mathrm{H}$, $\left.\mathrm{CH}_{2} \mathrm{OH}\right), 6.90(\mathrm{dd}, J=5.1,3.5 \mathrm{~Hz}, 1 \mathrm{H}, \mathrm{HetArH}), 7.01$ (s, 1H, NCH=), 7.02-7.06 (m, 2H, ArH), 7.15 (dd, $J=$ 5.1, $1.3 \mathrm{~Hz}, 1 \mathrm{H}, \mathrm{HetArH}), 7.17-7.23(\mathrm{~m}, 1 \mathrm{H}, \mathrm{ArH})$, 7.27-7.31 (m, 1H, ArH), 7.53-7.57 (m, 1H, ArH).

${ }^{13} \mathrm{C}$ NMR $\left(75.4 \mathrm{MHz}, \mathrm{CDCl}_{3}\right): \delta=31.8\left(\mathrm{CH}_{3}\right), 32.7$ $\left(\mathrm{CH}_{3}\right), 36.7(\mathrm{C}), 51.2\left(\mathrm{CH}_{2}\right), 80.3(\mathrm{C}), 90.6(\mathrm{C}), 109.5$ $(\mathrm{CH}), 119.0(\mathrm{CH}), 119.7(\mathrm{C}), 120.9(\mathrm{CH}), 121.7(\mathrm{CH})$, $124.1(\mathrm{CH}), 124.2(\mathrm{CH}), 125.6(\mathrm{C}), 126.2(\mathrm{CH}), 126.3$ (CH), $137.7(\mathrm{C}), 151.7(\mathrm{C})$.

MS (EI, $70 \mathrm{eV}): m / z(\%)=295\left(57, \mathrm{M}^{+}\right), 280(100)$, 264 (71).

HRMS-EI: $m / z$ [M] ${ }^{+}$calcd for $\mathrm{C}_{18} \mathrm{H}_{17} \mathrm{NOS}$ : 295.1031; found: 295.1034 .

2l isolated as a $\sim 1: 1$ mixture of diastereoisomers; white foam; yield: $437 \mathrm{mg}(66 \%) ; R_{f}=0.14$ (hexane- $\mathrm{Et}_{2} \mathrm{O}, 1.5: 1$ ).

${ }^{1} \mathrm{H}$ NMR $\left(300 \mathrm{MHz}, \mathrm{CDCl}_{3}\right): \delta=0.91(\mathrm{~d}, J=6.5 \mathrm{~Hz}$, $\left.3 \mathrm{H}, \mathrm{CHOHCHCH}_{3}\right), 1.26(\mathrm{~d}, J=6.5 \mathrm{~Hz}, 3 \mathrm{H}$, $\left.\mathrm{CH}_{3} \mathrm{CHCH}_{3}\right), 1.59\left(\mathrm{~d}, \mathrm{~J}=6.5 \mathrm{~Hz}, 3 \mathrm{H}, \mathrm{CH}_{3} \mathrm{CHCH}_{3}\right)$, 2.02 (bs, $1 \mathrm{H}, \mathrm{OH}$ ), 2.84 (sept, $J=6.5 \mathrm{~Hz}, 1 \mathrm{H}$, $\left.\mathrm{CH}_{3} \mathrm{CHCH}_{3}\right), 3.76\left(\mathrm{~s}, 3 \mathrm{H}, \mathrm{NCH}_{3}\right), 4.73(\mathrm{q}, J=6.5 \mathrm{~Hz}$, $1 \mathrm{H}, \mathrm{CHOH}), 6.98-7.03(\mathrm{~m}, 1 \mathrm{H}, \mathrm{ArH}), 7.12-7.20(\mathrm{~m}$, $3 \mathrm{H}, \mathrm{ArH}), 7.24-7.29$ (m, 3H, ArH), 7.61-7.64 (m, 2H, ArH), 7.66-7.71 (m, 1H, ArH). The signals of both isomers appear completely overlapped.

${ }^{13} \mathrm{C}$ NMR $\left(75.4 \mathrm{MHz}, \mathrm{CDCl}_{3}\right): \delta=19.0\left(\mathrm{CH}_{3}\right.$, both isomers), $20.2\left(\mathrm{CH}_{3}\right.$, both isomers $), 24.9\left(\mathrm{CH}_{3}\right.$, both isomers), $32.9\left(\mathrm{CH}_{3}\right.$, both isomers), $35.95(\mathrm{CH}), 35.97$ $(\mathrm{CH}), 50.0$ (C, both isomers), $58.9(\mathrm{CH}$, both isomers), 86.2 (C, both isomers), 88.1 (C, both isomers), 109.2 ( $\mathrm{CH}$, both isomers), 118.6 (C, both isomers), 118.8 ( $\mathrm{CH}$, both isomers), 121.46 ( $\mathrm{CH}$, both isomers), $121.48(\mathrm{CH}$, both isomers), $126.2(\mathrm{CH}$, both isomers), 126.6 (C, both isomers), 126.7 ( $\mathrm{CH}$, both isomers), $127.3(2 \times \mathrm{CH}$, both isomers $), 127.9(2 \times \mathrm{CH}$, both isomers), 137.5 (C, both isomers), 144.4 (C, both isomers).

MS (EI, $70 \mathrm{eV}): m / z(\%)=331\left(9, \mathrm{M}^{+}\right), 289(75), 288$ (100), 245 (55), 244 (39).

HRMS-EI: $m / z$ [M] $]^{+}$calcd for $\mathrm{C}_{23} \mathrm{H}_{25} \mathrm{NO}: 331.1936$; found: 331.1931 .

\section{Synthesis of 1-(3-cyclopropyl-2-(1-methyl-1H-} indol-3-yl)-1 $H$-inden-1-yl)butyl acetate (3a)

$\mathrm{PPh}_{3}$ AuNTf $_{2}(18.5 \mathrm{mg}, 5 \mathrm{~mol} \%$ ) was added to a solution of acetate 1a $(200 \mathrm{mg}, 0.5 \mathrm{mmol})$ in dry $\mathrm{CH}_{2} \mathrm{Cl}_{2}(1 \mathrm{~mL})$ and the mixture was stirred at room temperature until complete consumption of starting material (as evident by TLC or GC-MS analysis). The mixture was filtered through a pad of silica gel, the solvent was removed and the crude residue was purified by flash column chromatography on silica gel using hexane-EtOAc (20:1) as eluent to afford 3a as a $\sim$ 1:1 mixture of diastereoisomers; orange oil; yield: 62 $\operatorname{mg}(31 \%) ; R_{f}=0.25$ (hexane- $\mathrm{Et}_{2} \mathrm{O}, 2: 1$ ).

${ }^{1} \mathrm{H}$ NMR (300 MHz, $\mathrm{CDCl}_{3}$ ): $\delta=0.44-1.19$ (m, 22H both isomers, $\mathrm{CH}_{2} \mathrm{CH}_{2} \mathrm{CH}+\mathrm{CH}_{2} \mathrm{CH}_{2} \mathrm{CH}_{3}$ ), 1.44 (s, $3 \mathrm{H}, \mathrm{COCH}_{3}$ ), 1.87-2.02 (m, $2 \mathrm{H}$ both isomers, $\left.\mathrm{CH}_{2} \mathrm{CH}_{2} \mathrm{CH}\right), 2.22\left(\mathrm{~s}, 3 \mathrm{H}, \mathrm{COCH}_{3}\right), 3.88(\mathrm{~s}, 3 \mathrm{H}$, $\left.\mathrm{NCH}_{3}\right), 3.89\left(\mathrm{~s}, 3 \mathrm{H}, \mathrm{NCH}_{3}\right), 4.33-4.36(\mathrm{~m}, 1 \mathrm{H}$, CHCHOAc), $4.45-4.49$ (m, $1 \mathrm{H}, \quad \mathrm{CHCHOAc})$, 5.10-5.17 (m, 1H, CHOAc), 5.30-5.37 (m, $1 \mathrm{H}$, CHOAc), 7.10-7.40 (m, 12H both isomers, $\mathrm{ArH})$, 7.45-7.59 (m, 4H both isomers, ArH), 7.63-7.67 (m, $1 \mathrm{H}, \mathrm{ArH}), 7.85-7.90$ (m, 1H, ArH).

${ }^{13} \mathrm{C}$ NMR $\left(75.4 \mathrm{MHz}, \mathrm{CDCl}_{3}\right): \delta=5.6\left(\mathrm{CH}_{2}\right), 5.9$ $\left(\mathrm{CH}_{2}\right), 6.8\left(\mathrm{CH}_{2}\right), 6.9\left(\mathrm{CH}_{2}\right), 9.2(\mathrm{CH}), 9.8(\mathrm{CH}), 13.7$ $\left(\mathrm{CH}_{3}\right.$, both isomers), $19.0\left(\mathrm{CH}_{2}\right), 19.4\left(\mathrm{CH}_{2}\right), 20.9$ $\left(\mathrm{CH}_{3}\right), 21.6\left(\mathrm{CH}_{3}\right), 29.6\left(\mathrm{CH}_{2}\right), 30.5\left(\mathrm{CH}_{2}\right), 33.1$ $\left(\mathrm{CH}_{3}\right), 33.2\left(\mathrm{CH}_{3}\right), 54.1(\mathrm{CH}), 54.6(\mathrm{CH}), 75.8(\mathrm{CH})$, $76.5(\mathrm{CH}), 109.3(\mathrm{CH}$, both isomers), $110.6(\mathrm{C}), 112.0$ (C), $119.5(\mathrm{CH}), 119.6(\mathrm{CH}), 119.78(\mathrm{CH}), 119.79$ $(\mathrm{CH}), 120.8(\mathrm{CH}), 121.5(\mathrm{CH}), 121.6(\mathrm{CH}), 121.8$ $(\mathrm{CH}), 123.2(\mathrm{CH}), 124.0(\mathrm{CH}), 124.4(\mathrm{CH}$, both isomers), $126.95(\mathrm{CH}), 127.00(\mathrm{CH}), 127.6(\mathrm{C}), 128.4$ (C), $128.7(\mathrm{CH}), 129.0(\mathrm{CH}), 136.9(\mathrm{C}), 137.0(\mathrm{C})$, 137.1 (C), 137.9 (C, both isomers), 139.4 (C), 143.3 (C), 143.5 (C), 146.5 (C), 147.4 (C), 171.2 (C), 171.3 (C).

MS (EI, $70 \mathrm{eV}): \mathrm{m} / z(\%)=399\left(54, \mathrm{M}^{+}\right), 339(37)$, 296 (46), 284 (100).

HRMS-EI: $m / z$ [M] $]^{+}$calcd for $\mathrm{C}_{27} \mathrm{H}_{29} \mathrm{NO}_{2}$ : 339.2198; found: 339.2197 .

Gold-catalyzed Reaction of Alkynols 2; 3-((11E)-1butylidene-3-cyclopropyl-1H-inden-2-yl)-1-methyl$1 H$-indole (5a) and 1-(3-cyclopropyl-2-(1-methyl$1 H$-indol-3-yl)-1 $H$-inden-1-yl)butan-1-ol Typical Procedure

$(\mathrm{PhO})_{3} \mathrm{PAuCl}(13.6 \mathrm{mg}, 5 \mathrm{~mol} \%)$ and $\mathrm{AgNTf}_{2}(9.7$ $\mathrm{mg}, 5 \mathrm{~mol} \%$ ) were successively added to a solution of alkynol $2 \mathbf{a}(179 \mathrm{mg}, 0.5 \mathrm{mmol})$ in dry $\mathrm{CH}_{2} \mathrm{Cl}_{2}(1 \mathrm{~mL})$ at $-78{ }^{\circ} \mathrm{C}$. The mixture was stirred at low temperature until complete consumption of starting material (as evident by TLC or GC-MS analysis). The mixture was filtered through a pad of silica gel, the solvent was removed and the crude residue was purified by flash column chromatography on silica gel using hexane-EtOAc (20:1) as eluent to afford 5a and then hexane-EtOAc $(4: 1)$ as eluent to afford $\mathbf{4 a}$.

5a, yellow oil; yield: $127 \mathrm{mg}(75 \%) ; R_{f}=0.23$ (hexane-EtOAc, 20:1).

${ }^{1} \mathrm{H}$ NMR $\left(300 \mathrm{MHz}, \mathrm{CDCl}_{3}\right): \delta=0.67-0.82(\mathrm{~m}, 4 \mathrm{H}$, $\left.\mathrm{CH}_{2} \mathrm{CH}_{2} \mathrm{CH}\right), 1.06\left(\mathrm{t}, J=7.4 \mathrm{~Hz}, 3 \mathrm{H}, \mathrm{CH}_{3} \mathrm{CH}_{2}\right)$, $1.54-1.73\left(\mathrm{~m}, 2 \mathrm{H}, \mathrm{CH}_{3} \mathrm{CH}_{2}\right), 1.88-2.02(\mathrm{~m}, 1 \mathrm{H}$, $\mathrm{CH}_{2} \mathrm{CH}_{2} \mathrm{CH}$ ), 2.83 (q, $J=7.5 \mathrm{~Hz}, 2 \mathrm{H},=\mathrm{CHCH}_{2}$ ), 3.92 (s, $\left.3 \mathrm{H}, \mathrm{NCH}_{3}\right), 6.31(\mathrm{t}, J=7.5 \mathrm{~Hz}, 1 \mathrm{H}, \mathrm{HC}=\mathrm{C}), 7.08$ (s, $1 \mathrm{H}, \mathrm{NCH}=), 7.15-7.23(\mathrm{~m}, 1 \mathrm{H}, \mathrm{ArH}), 7.24-7.52$ (m, 5H, ArH), 7.64-7.71 (m, 1H, ArH), 7.83-7.90 (m, $1 \mathrm{H}, \mathrm{ArH})$. 
${ }^{13} \mathrm{C} \mathrm{NMR}\left(75.4 \mathrm{MHz}, \mathrm{CDCl}_{3}\right): \delta=5.9\left(2 \times \mathrm{CH}_{2}\right), 9.9$ $(\mathrm{CH}), 14.3\left(\mathrm{CH}_{3}\right), 23.0\left(\mathrm{CH}_{2}\right), 31.8\left(\mathrm{CH}_{2}\right), 33.0\left(\mathrm{CH}_{3}\right)$, $109.2(\mathrm{CH}), 109.4(\mathrm{C}), 119.3(\mathrm{CH}), 119.4(\mathrm{CH}), 121.2$ $(\mathrm{CH}), 121.7(\mathrm{CH}), 123.4(\mathrm{CH}), 124.5(\mathrm{CH}), 127.1$ $(\mathrm{CH}), 129.0(\mathrm{C}), 129.1(\mathrm{CH}), 132.7(\mathrm{C}), 135.8(\mathrm{C})$, $136.0(\mathrm{CH}), 136.8(\mathrm{C}), 139.0(\mathrm{C}), 140.4(\mathrm{C}), 144.5$ (C).

MS (EI, $70 \mathrm{eV}): m / z(\%)=339\left(100, \mathrm{M}^{+}\right), 310(34)$, 296 (52), 281 (27), 268 (30).

HRMS-EI: $m / z$ [M] $]^{+}$calcd for $\mathrm{C}_{25} \mathrm{H}_{25} \mathrm{~N}$ : 339.1987; found: 339.1982 .

4a, isolated in the reaction of $\mathbf{2 a}$ with $\mathrm{PPh}_{3} \mathrm{AuNTf}_{2}$ at r.t. in $36 \%$ yield $(64 \mathrm{mg})$ and as a $\sim 1.5: 1$ mixture of diastereoisomers, brown oil; $R_{f}=0.10$ (hexane-EtOAc, 6:1).

${ }^{1} \mathrm{H}$ NMR $\left(300 \mathrm{MHz}, \mathrm{CDCl}_{3}\right): \delta=0.44-0.97(\mathrm{~m}, 7 \mathrm{H}$ maj $+7 \mathrm{H}$ min, $\mathrm{CH}_{2} \mathrm{CH}_{2} \mathrm{CH}+\mathrm{CH}_{2} \mathrm{CH}_{2} \mathrm{CH}_{3}$ ), 1.17-1.58 (m, 4H maj $+4 \mathrm{H}$ min, $\left.\mathrm{CH}_{2} \mathrm{CH}_{2} \mathrm{CH}_{3}\right)$, 1.83-1.96 (m, $1 \mathrm{H}$ maj $+1 \mathrm{H}$ min, $\left.\mathrm{CH}_{2} \mathrm{CH}_{2} \mathrm{CH}\right), 3.85$ (s, 3H maj, $\left.\mathrm{NCH}_{3}\right), 3.86$ (s, 3H min, $\mathrm{NCH}_{3}$ ), 3.96-3.99 (m, 1H maj, CHOH), 4.00-4.05 (m, 1H $\min , \mathrm{CHOH}), 4.22(\mathrm{bs}, 1 \mathrm{H} \min , \mathrm{CHCHOH}), 4.31(\mathrm{~d}, J$ $=4.1 \mathrm{~Hz}, 1 \mathrm{H}$ maj, $\mathrm{CHCHOH}), 7.13-7.51(\mathrm{~m}, 7 \mathrm{H}$ maj $+8 \mathrm{H} \mathrm{min}, \mathrm{ArH}), 7.75$ (d, $J=8.0 \mathrm{~Hz}, 1 \mathrm{H} \mathrm{maj}, \mathrm{ArH})$, $7.67(\mathrm{~d}, J=7.4 \mathrm{~Hz}, 1 \mathrm{H} \mathrm{min}, \mathrm{ArH}), 7.80(\mathrm{~d}, J=8.0 \mathrm{~Hz}$, $1 \mathrm{H}$ maj, $\mathrm{ArH})$. The signal corresponding to the $\mathrm{OH}$ is not observed.

${ }^{13} \mathrm{C}$ NMR $\left(75.4 \mathrm{MHz}, \mathrm{CDCl}_{3}\right): \delta=5.68\left(\mathrm{CH}_{2}, \mathrm{~min}\right)$, 5.71 $\left(\mathrm{CH}_{2}\right.$, maj), $7.0\left(\mathrm{CH}_{2}, \mathrm{~min}\right), 7.1\left(\mathrm{CH}_{2}\right.$, maj $), 9.2$ $\left(\mathrm{CH}\right.$, both isomers), $13.9\left(\mathrm{CH}_{3}, \mathrm{maj}\right), 14.2\left(\mathrm{CH}_{3}, \mathrm{~min}\right)$, $19.2\left(\mathrm{CH}_{2}, \mathrm{maj}\right), 19.9\left(\mathrm{CH}_{2}, \mathrm{~min}\right), 33.0\left(\mathrm{CH}_{2}, \mathrm{maj}\right)$, $33.1\left(\mathrm{CH}_{3}, \mathrm{maj}\right), 33.2\left(\mathrm{CH}_{3}, \mathrm{~min}\right), 36.6\left(\mathrm{CH}_{2}, \mathrm{~min}\right)$, $57.4(\mathrm{CH}, \mathrm{min}), 57.9(\mathrm{CH}, \mathrm{maj}), 73.1(\mathrm{CH}, \mathrm{min}), 73.6$ (CH, maj), 109.5 (CH, maj), 109.6 (CH, min), 111.1 (C, both isomers), $119.71(\mathrm{CH}$, maj), $119.74(\mathrm{CH}$, maj), $119.85(\mathrm{CH}, \mathrm{min}), 119.94(\mathrm{CH}$, maj), $120.8(\mathrm{CH}$, maj), $120.9(\mathrm{CH}, \mathrm{min}), 121.9(\mathrm{CH}, \mathrm{maj}), 122.0(\mathrm{CH}$, $\min ), 123.8(\mathrm{CH}, \mathrm{min}), 124.1(\mathrm{CH}, \mathrm{maj}), 124.2(\mathrm{CH}$, $\min ), 124.4(\mathrm{CH}, \mathrm{maj}), 126.8(\mathrm{CH}, \mathrm{maj}), 127.1(\mathrm{CH}$, $\min ), 127.5(\mathrm{C}, \mathrm{min}), 127.6(\mathrm{C}, \mathrm{maj}), 128.9(\mathrm{CH}$, maj), 129.1 ( $\mathrm{CH}, \mathrm{min}), 137.0$ (C, maj), 137.1 (C, min), 137.6 (C, maj), 138.0 (C, maj), 138.1 (C, min), 138.9 (C, min), 142.7 (C, min), 144.2 (C, maj), 147.1 (C, maj), 147.2 (C, min).

5b, yellow solid; yield: $114 \mathrm{mg}(77 \%) ; R_{f}=0.28$ (hexane- $\left.\mathrm{Et}_{2} \mathrm{O}, 1.5: 1\right) ; \mathrm{mp}=128^{\circ} \mathrm{C}$ (decomposition).

${ }^{1} \mathrm{H}$ NMR $\left(300 \mathrm{MHz}, \mathrm{CDCl}_{3}\right): \delta=0.73-0.94(\mathrm{~m}, 4 \mathrm{H}$, $\left.\mathrm{CH}_{2} \mathrm{CH}_{2} \mathrm{CH}\right), 1.93-2.04\left(\mathrm{~m}, 1 \mathrm{H}, \mathrm{CH}_{2} \mathrm{CH}_{2} \mathrm{CH}\right), 3.89$ $\left(\mathrm{s}, 3 \mathrm{H}, \mathrm{NCH}_{3}\right), 5.61(\mathrm{~s}, 1 \mathrm{H}, H \mathrm{HC}=\mathrm{C}), 6.02(\mathrm{~s}, 1 \mathrm{H}$, $\mathrm{H} H \mathrm{C}=\mathrm{C}), 7.09(\mathrm{~s}, 1 \mathrm{H}, \mathrm{NCH}=), 7.10-7.17(\mathrm{~m}, 1 \mathrm{H}$, ArH $), 7.18-7.32(\mathrm{~m}, 4 \mathrm{H}, \operatorname{ArH}), 7.37-7.41(\mathrm{~m}, 1 \mathrm{H}$, ArH), 7.62-7.69 (m, 2H, ArH).

${ }^{13} \mathrm{C}$ NMR $\left(75.4 \mathrm{MHz}, \mathrm{CDCl}_{3}\right): \delta=6.1\left(2 \times \mathrm{CH}_{2}\right), 10.8$ $(\mathrm{CH}), 33.0\left(\mathrm{CH}_{3}\right), 109.0(\mathrm{C}), 109.3(\mathrm{CH}), 111.3\left(\mathrm{CH}_{2}\right)$, $119.4(2 \times \mathrm{CH}), 119.5(\mathrm{CH}), 121.2(\mathrm{CH}), 121.8(\mathrm{CH})$,
$124.9(\mathrm{CH}), 128.1(\mathrm{CH}), 128.5(\mathrm{C}), 128.9(\mathrm{CH}), 130.9$ (C), 136.8 (C), 136.9 (C), 142.7 (C), 143.1 (C), 147.9 (C).

MS (EI, $70 \mathrm{eV}): m / z(\%)=297\left(100, \mathrm{M}^{+}\right), 296(64)$, 282 (28), 268 (23).

HRMS-EI: $m / z$ [M] ${ }^{+}$calcd for $\mathrm{C}_{22} \mathrm{H}_{19} \mathrm{~N}: 297.1517$; found: 297.1517 .

5c, orange solid; yield: $163 \mathrm{mg}(80 \%) ; R_{f}=0.26$ (hexane- $\mathrm{Et}_{2} \mathrm{O}, 15: 1$ ); $\mathrm{mp}=124-126^{\circ} \mathrm{C}$.

${ }^{1} \mathrm{H}$ NMR $\left(300 \mathrm{MHz}, \mathrm{CDCl}_{3}\right): \delta=0.65-0.95(\mathrm{~m}, 4 \mathrm{H}$, $\mathrm{CH}_{2} \mathrm{CH}_{2} \mathrm{CH}$ ), 1.91-1.99 (m, $\left.1 \mathrm{H}, \mathrm{CH}_{2} \mathrm{CH}_{2} \mathrm{CH}\right), 3.91$ (s, 3H, $\left.\mathrm{NCH}_{3}\right), 6.98-7.08(\mathrm{~m}, 2 \mathrm{H}, \mathrm{ArH}), 7.11-7.23$ (m, 2H, ArH), 7.24-7.52 (m, 9H, ArH), 7.66-7.72 (m, $1 \mathrm{H}, \mathrm{ArH})$.

${ }^{13} \mathrm{C}$ NMR $\left(75.4 \mathrm{MHz}, \mathrm{CDCl}_{3}\right): \delta=6.1\left(2 \times \mathrm{CH}_{2}\right), 10.4$ $(\mathrm{CH}), 33.1\left(\mathrm{CH}_{3}\right), 109.0(\mathrm{C}), 109.4(\mathrm{CH}), 119.5(\mathrm{CH})$, $119.6(\mathrm{CH}), 121.1(\mathrm{CH}), 121.9(\mathrm{CH}), 122.9(\mathrm{CH})$, $124.7(\mathrm{CH}), 128.1(\mathrm{CH}), 128.6(2 \times \mathrm{CH}), 128.9(\mathrm{C})$, $129.3(\mathrm{CH}), 130.1(\mathrm{CH}), 130.9(2 \times \mathrm{CH}), 132.7(\mathrm{C})$, 133.6 (C), 134.9 (C), 136.1 (C), 136.9 (C), 141.7 (C), 142.1 (C), 144.6 (C).

MS (EI, $70 \mathrm{eV}): \mathrm{m} / \mathrm{z}(\%)=407\left(100, \mathrm{M}^{+}\right), 406(27)$, 296 (37), 282 (29).

HRMS-EI: $m / z$ [M] ${ }^{+}$calcd for $\mathrm{C}_{28} \mathrm{H}_{22} \mathrm{ClN}$ : 407.1441; found: 407.1443 .

5d, yellow solid; yield: $131 \mathrm{mg}(84 \%) ; R_{f}=0.32$ (hexane- $\left.\mathrm{Et}_{2} \mathrm{O}, 10: 1\right) ; \mathrm{mp}=58-60^{\circ} \mathrm{C}$.

${ }^{1} \mathrm{H}$ NMR $\left(300 \mathrm{MHz}, \mathrm{CDCl}_{3}\right): \delta=0.63-0.79(\mathrm{~m}, 4 \mathrm{H}$, $\mathrm{CH}_{2} \mathrm{CH}_{2} \mathrm{CH}$ ), 1.91 (quint, $J=7.1 \mathrm{~Hz}, 1 \mathrm{H}$, $\left.\mathrm{CH}_{2} \mathrm{CH}_{2} \mathrm{CH}\right), 2.33\left(\mathrm{~d}, J=7.6 \mathrm{~Hz}, 3 \mathrm{H},=\mathrm{CHCH}_{3}\right), 3.89$ $\left(\mathrm{s}, 3 \mathrm{H}, \mathrm{NCH}_{3}\right), 6.33\left(\mathrm{q}, J=7.6 \mathrm{~Hz}, 1 \mathrm{H},=\mathrm{CHCH}_{3}\right)$, $7.05(\mathrm{~s}, 1 \mathrm{H}, \mathrm{NCH}=), 7.11-7.18(\mathrm{~m}, 1 \mathrm{H}, \mathrm{ArH})$, 7.21-7.47 (m, 5H, ArH), 7.59-7.63 (m, 1H, ArH), 7.82-7.86 (m, 1H, ArH).

${ }^{13} \mathrm{C}$ NMR $\left(75.4 \mathrm{MHz}, \mathrm{CDCl}_{3}\right): \delta=5.9\left(2 \times \mathrm{CH}_{2}\right), 9.9$ $(\mathrm{CH}), 15.7\left(\mathrm{CH}_{3}\right), 33.0\left(\mathrm{CH}_{3}\right), 109.2(\mathrm{CH}), 109.4(\mathrm{C})$, $119.3(\mathrm{CH}), 119.4(\mathrm{CH}), 121.1(\mathrm{CH}), 121.7(\mathrm{CH})$, $123.5(\mathrm{CH}), 124.5(\mathrm{CH}), 127.1(\mathrm{CH}), 128.9(\mathrm{C}), 129.1$ $(\mathrm{CH}), 129.8(\mathrm{CH}), 132.5(\mathrm{C}), 136.0(\mathrm{C}), 136.8(\mathrm{C})$, 139.0 (C), 141.5 (C), 144.5 (C).

MS (EI, $70 \mathrm{eV}): m / z(\%)=311\left(100, \mathrm{M}^{+}\right), 296(61)$, 281 (34), 268 (21).

HRMS-EI: $m / z[\mathrm{M}]^{+}$calcd for $\mathrm{C}_{23} \mathrm{H}_{21} \mathrm{~N}: 311.1674$; found: 311.1678 .

5e, yellow solid; yield: $52 \mathrm{mg}(32 \%) ; R_{f}=0.29$ (hexane- $\mathrm{Et}_{2} \mathrm{O}, 15: 1$ ); $\mathrm{mp}=165-167^{\circ} \mathrm{C}$.

${ }^{1} \mathrm{H}$ NMR $\left(300 \mathrm{MHz}, \mathrm{CDCl}_{3}\right): \delta=0.49-0.71(\mathrm{~m}, 4 \mathrm{H}$, $\left.\mathrm{CH}_{2} \mathrm{CH}_{2} \mathrm{CH}\right), 1.69$ (s, $\left.3 \mathrm{H}, \mathrm{CH}_{3} \mathrm{CCH}_{3}\right), 1.68-1.77$ (m, $\left.1 \mathrm{H}, \mathrm{CH}_{2} \mathrm{CH}_{2} \mathrm{CH}\right), 2.42\left(\mathrm{~s}, 3 \mathrm{H}, \mathrm{CH}_{3} \mathrm{CCH}_{3}\right), 3.86(\mathrm{~s}$, $\left.3 \mathrm{H}, \mathrm{NCH}_{3}\right), 6.94(\mathrm{~s}, 1 \mathrm{H}, \mathrm{NCH}=), 7.04-7.10(\mathrm{~m}, 1 \mathrm{H}$, ArH), 7.17-7.26 (m, 3H, ArH), 7.32-7.36 (m, 1H, 
ArH), 7.39-7.44 (m, 1H, ArH), 7.78-7.81 (m, 1H, ArH).

${ }^{13} \mathrm{C}$ NMR $\left(75.4 \mathrm{MHz}, \mathrm{CDCl}_{3}\right): \delta=5.5\left(\mathrm{CH}_{2}\right), 6.2$ $\left(\mathrm{CH}_{2}\right), 9.5(\mathrm{CH}), 24.9\left(\mathrm{CH}_{3}\right), 26.1\left(\mathrm{CH}_{3}\right), 32.9\left(\mathrm{CH}_{3}\right)$, $109.1(\mathrm{CH}), 113.5(\mathrm{C}), 119.3(\mathrm{CH}), 119.4(\mathrm{CH}), 120.7$ $(\mathrm{CH}), 121.7(\mathrm{CH}), 123.9(\mathrm{CH}), 124.5(\mathrm{CH}), 125.8$ (CH), $127.8(\mathrm{CH}), 129.3(\mathrm{C}), 131.8(\mathrm{C}), 136.5(\mathrm{C})$, 137.0 (C), 137.5 (C), 141.5 (C), 142.8 (C), 142.9 (C).

MS (EI, $70 \mathrm{eV}): m / z(\%)=325\left(100, \mathrm{M}^{+}\right), 310(79)$, 295 (27), 282 (33).

HRMS-EI: $m / z[\mathrm{M}]^{+}$calcd for $\mathrm{C}_{24} \mathrm{H}_{23} \mathrm{~N}$ : 325.1830 ; found: 325.1825 .

4e, white solid; yield: $89 \mathrm{mg}(52 \%) ; R_{f}=0.14$ (hexane- $\mathrm{Et}_{2} \mathrm{O}, 1: 2$ ); $\mathrm{mp}=72-74^{\circ} \mathrm{C}$.

${ }^{1} \mathrm{H}$ NMR (300 MHz, $\left.\mathrm{CDCl}_{3}\right): \delta=0.34-0.44(\mathrm{~m}, 2 \mathrm{H}$, $\left.\mathrm{CH}_{2} \mathrm{CH}_{2} \mathrm{CH}\right), 0.57-0.64\left(\mathrm{~m}, 1 \mathrm{H}, \mathrm{CH}_{2} \mathrm{CHHCH}\right), 0.79$ (s, $\left.3 \mathrm{H}, \mathrm{CH}_{3} \mathrm{CCH}_{3}\right), 0.82-0.94\left(\mathrm{~m}, 1 \mathrm{H}, \mathrm{CH}_{2} \mathrm{CH} H \mathrm{CH}\right)$, $1.32\left(\mathrm{~s}, \quad 3 \mathrm{H}, \quad \mathrm{CH}_{3} \mathrm{CCH}_{3}\right), \quad 1.82-1.92(\mathrm{~m}, \quad 1 \mathrm{H}$, $\mathrm{CH}_{2} \mathrm{CH}_{2} \mathrm{CH}$ ), 2.04 (bs, $\left.1 \mathrm{H}, \mathrm{OH}\right), 3.86$ (s, $3 \mathrm{H}, \mathrm{NCH}_{3}$ ), 4.08 (bs, $1 \mathrm{H}, \mathrm{CHCOH}), 7.14(\mathrm{~s}, 1 \mathrm{H}, \mathrm{NCH}=)$, 7.15-7.19 (m, 2H, ArH), 7.25-7.37 (m, 3H, ArH), 7.45-7.50 (m, 1H, ArH), 7.59-7.64 (m, 2H, ArH), 7.72-7.78 (m, 1H, ArH).

${ }^{13} \mathrm{C}$ NMR $\left(75.4 \mathrm{MHz}, \mathrm{CDCl}_{3}\right): \delta=5.2\left(\mathrm{CH}_{2}\right), 6.5$ $\left(\mathrm{CH}_{2}\right), 9.4(\mathrm{CH}), 25.9\left(\mathrm{CH}_{3}\right), 28.3\left(\mathrm{CH}_{3}\right), 33.1\left(\mathrm{CH}_{3}\right)$, $63.2(\mathrm{CH}), 74.4(\mathrm{C}), 109.5(\mathrm{CH}), 112.1(\mathrm{C}), 119.8$ $(\mathrm{CH}), 119.9(\mathrm{CH}), 121.3(\mathrm{CH}), 122.2(\mathrm{CH}), 124.1$ $(\mathrm{CH}), 124.9(\mathrm{CH}), 126.9(\mathrm{CH}), 127.5(\mathrm{C}), 129.0(\mathrm{CH})$, 137.0 (C), 138.0 (C), 140.1 (C), 144.2 (C), 146.6 (C).

MS (EI, $70 \mathrm{eV}): m / z(\%)=343\left(17, \mathrm{M}^{+}\right), 285(100)$, 270 (23), 256 (15).

HRMS-EI: $m / z$ [M] $]^{+}$calcd for $\mathrm{C}_{24} \mathrm{H}_{25} \mathrm{NO}$ : 343.1936 ; found: 343.1930 .

4f, pale orange solid; yield: $187 \mathrm{mg}(70 \%) ; R_{f}=0.24$ (hexane- $\mathrm{Et}_{2} \mathrm{O}, 3: 1$ ); $\mathrm{mp}=187-189^{\circ} \mathrm{C}$.

${ }^{1} \mathrm{H}$ NMR $\left(300 \mathrm{MHz}, \mathrm{CDCl}_{3}\right): \delta=0.28-0.39(\mathrm{~m}, 2 \mathrm{H}$, $\left.\mathrm{CH}_{2} \mathrm{CH}_{2} \mathrm{CH}\right), \quad 0.51-0.58\left(\mathrm{~m}, \quad 1 \mathrm{H}, \quad \mathrm{CH}_{2} \mathrm{CH} \mathrm{HCH}\right)$, 0.70-0.79 (m, 1H, $\left.\mathrm{CH}_{2} \mathrm{CH} H \mathrm{CH}\right), 1.56-1.65(\mathrm{~m}, 1 \mathrm{H}$, $\left.\mathrm{CH}_{2} \mathrm{CH}_{2} \mathrm{CH}\right), 3.21$ (bs, $\left.1 \mathrm{H}, \mathrm{OH}\right), 3.75\left(\mathrm{~s}, 3 \mathrm{H}, \mathrm{NCH}_{3}\right)$, 4.73 (bs, $1 \mathrm{H}, \mathrm{CHCOH}), 6.04(\mathrm{~d}, J=7.6 \mathrm{~Hz}, 1 \mathrm{H}, \mathrm{ArH})$, 6.63 (bs, 1H, ArH), 6.79 (d, $J=8.5 \mathrm{~Hz}, 2 \mathrm{H}, \mathrm{ArH})$, 6.89-6.95 (m, 1H, ArH), 7.04-7.31 (m, 11H, ArH), $7.42(\mathrm{~d}, J=7.6 \mathrm{~Hz}, 1 \mathrm{H}, \mathrm{ArH})$

${ }^{13} \mathrm{C}$ NMR $\left(75.4 \mathrm{MHz}, \mathrm{CDCl}_{3}\right): \delta=4.9\left(\mathrm{CH}_{2}\right), 6.0$ $\left(\mathrm{CH}_{2}\right), 9.2(\mathrm{CH}), 33.0\left(\mathrm{CH}_{3}\right), 63.4(\mathrm{CH}), 80.4(\mathrm{C})$, 109.4 (CH), $111.0(\mathrm{C}), 119.9(\mathrm{CH}), 120.1(\mathrm{CH}), 120.7$ $(\mathrm{CH}), 122.3(\mathrm{CH}), 124.6(\mathrm{CH}), 124.8(\mathrm{CH}), 127.5$ $(\mathrm{CH}), 127.7(2 \times \mathrm{CH}), 127.8(2 \times \mathrm{CH}), 128.0(2 \times$ $\mathrm{CH}), 128.6(\mathrm{CH}), 128.9(2 \times \mathrm{CH}), 133.0(\mathrm{C}), 133.2$ (C), 136.8 (C), 136.9 (C), 142.1 (C), 142.6 (C), 142.7 $\begin{array}{llll}\text { (C), } 143.8 & \text { (C), } 146.5 & \text { (C). The signal of one }\end{array}$ quaternary aromatic carbon is not observed, probably overlapped by other signal.
MS (EI, $70 \mathrm{eV}): \mathrm{m} / z(\%)=285$ (100), $270(16), 139$ (43). $[\mathrm{M}]^{+}$not observed.

HRMS-EI: $m / z \quad[\mathrm{M}]^{+}$calcd for $\mathrm{C}_{34} \mathrm{H}_{27} \mathrm{Cl}_{2} \mathrm{NO}$ : 535.1470; found: 535.1467.

5g, orange solid; yield: $74 \mathrm{mg}(45 \%) ; R_{f}=0.20$ (hexane- $\left.\mathrm{Et}_{2} \mathrm{O}, 10: 1\right) ; \mathrm{mp}=200-202^{\circ} \mathrm{C}$.

${ }^{1} \mathrm{H}$ NMR $\left(300 \mathrm{MHz}, \mathrm{CDCl}_{3}\right): \delta=0.74-0.83(\mathrm{~m}, 2 \mathrm{H}$, $\left.\mathrm{CH}_{2} \mathrm{CH}_{2} \mathrm{CH}\right), \quad 0.94-0.99\left(\mathrm{~m}, \quad 2 \mathrm{H}, \quad \mathrm{CH}_{2} \mathrm{CH}_{2} \mathrm{CH}\right)$, $1.93-2.03\left(\mathrm{~m}, 1 \mathrm{H}, \mathrm{CH}_{2} \mathrm{CH}_{2} \mathrm{CH}\right), 3.88\left(\mathrm{~s}, 3 \mathrm{H}, \mathrm{NCH}_{3}\right.$ or $\left.\mathrm{OCH}_{3}\right), 3.89\left(\mathrm{~s}, 3 \mathrm{H}, \mathrm{NCH}_{3}\right), 5.58(\mathrm{~s}, 1 \mathrm{H}, H \mathrm{HC}=\mathrm{C})$, $5.98(\mathrm{~s}, 1 \mathrm{H}, \mathrm{H} H \mathrm{C}=\mathrm{C}), 6.81-6.87(\mathrm{~m}, 1 \mathrm{H}, \mathrm{ArH}), 7.09$ (s, 1H, NCH=), 7.11-7.22 (m, 2H, ArH), 7.24-7.32 (m, 2H, ArH), 7.37-7.41 (m, 1H, ArH), 7.65-7.70 (m, $1 \mathrm{H}, \mathrm{ArH})$.

${ }^{13} \mathrm{C} \mathrm{NMR}\left(75.4 \mathrm{MHz}, \mathrm{CDCl}_{3}\right): \delta=6.0\left(2 \times \mathrm{CH}_{2}\right), 11.1$ $(\mathrm{CH}), 33.0\left(\mathrm{CH}_{3}\right), 55.8\left(\mathrm{CH}_{3}\right), 106.6(\mathrm{CH}), 109.1(\mathrm{C})$, $109.3(\mathrm{CH}), 110.7\left(\mathrm{CH}_{2}\right), 112.8(\mathrm{CH}), 119.3(\mathrm{CH})$, $119.8(\mathrm{CH}), 121.2(\mathrm{CH}), 121.7(\mathrm{CH}), 128.5(\mathrm{C}), 128.8$ (CH), 129.4 (C), 136.1 (C), 136.9 (C), 138.9 (C), 142.9 (C), 147.9 (C), $158.2(\mathrm{C})$.

MS (EI, $70 \mathrm{eV}): m / z(\%)=327\left(100, \mathrm{M}^{+}\right), 326(35)$, 312 (28).

HRMS-EI: $m / z[\mathrm{M}]^{+}$calcd for $\mathrm{C}_{23} \mathrm{H}_{21} \mathrm{NO}$ : 327.1623; found: 327.1614 .

5h, orange solid; yield: $111 \mathrm{mg}(51 \%) ; R_{f}=0.25$ (hexane- $\mathrm{Et}_{2} \mathrm{O}, 10: 1$ ); $\mathrm{mp}=160-162^{\circ} \mathrm{C}$.

${ }^{1} \mathrm{H}$ NMR $\left(300 \mathrm{MHz}, \mathrm{CDCl}_{3}\right): \delta=0.62-0.91(\mathrm{~m}, 4 \mathrm{H}$, $\left.\mathrm{CH}_{2} \mathrm{CH}_{2} \mathrm{CH}\right), 1.85-1.97\left(\mathrm{~m}, 1 \mathrm{H}, \mathrm{CH}_{2} \mathrm{CH}_{2} \mathrm{CH}\right), 3.68$ $\left(\mathrm{s}, 3 \mathrm{H}, \mathrm{OCH}_{3}\right), 3.89\left(\mathrm{~s}, 3 \mathrm{H}, \mathrm{NCH}_{3}\right), 6.75-6.81(\mathrm{~m}, 1 \mathrm{H}$, $\mathrm{ArH}), 6.99$ (s, 1H, NCH=), 7.06-7.44 (m, 10H, ArH), 7.62-7.67 (m, 1H, ArH).

${ }^{13} \mathrm{C} \mathrm{NMR}\left(75.4 \mathrm{MHz}, \mathrm{CDCl}_{3}\right): \delta=6.0\left(2 \times \mathrm{CH}_{2}\right), 10.6$ $(\mathrm{CH}), 33.1\left(\mathrm{CH}_{3}\right), 55.6\left(\mathrm{CH}_{3}\right), 109.1(\mathrm{C}), 109.3(\mathrm{CH})$, $110.3(\mathrm{CH}), 112.6(\mathrm{CH}), 119.5(\mathrm{CH}), 119.7(\mathrm{CH})$, $121.1(\mathrm{CH}), 121.8(\mathrm{CH}), 128.7(2 \times \mathrm{CH}), 128.9(\mathrm{C})$, $129.2(\mathrm{CH}), 129.7(\mathrm{CH}), 130.7(2 \times \mathrm{CH}), 131.0(\mathrm{C})$, 133.7 (C), 136.1 (C), 136.6 (C), 136.9 (C), 137.6 (C), 141.8 (C), 142.1 (C), 157.7 (C).

MS (EI, $70 \mathrm{eV}): m / z(\%)=437\left(100, \mathrm{M}^{+}\right), 422(15)$, 326 (18), 312 (15).

HRMS-EI: $m / z \quad[\mathrm{M}]^{+}$calcd for $\mathrm{C}_{29} \mathrm{H}_{24} \mathrm{CINO}$ : 437.1546; found: 437.1543 .

5i, yellow solid; yield: $135 \mathrm{mg}(78 \%) ; R_{f}=0.29$ (hexane- $\mathrm{Et}_{2} \mathrm{O}, 10: 1$ ); $\mathrm{mp}=61-63^{\circ} \mathrm{C}$.

${ }^{1} \mathrm{H}$ NMR $\left(300 \mathrm{MHz}, \mathrm{CDCl}_{3}\right): \delta=0.61-0.79(\mathrm{~m}, 4 \mathrm{H}$, $\mathrm{CH}_{2} \mathrm{CH}_{2} \mathrm{CH}$ ), 1.81-1.92 (m, $1 \mathrm{H}, \mathrm{CH}_{2} \mathrm{CH}_{2} \mathrm{CH}$ ), 2.30 $\left(\mathrm{d}, J=7.6 \mathrm{~Hz}, 3 \mathrm{H},=\mathrm{CHCH}_{3}\right), 3.89\left(\mathrm{~s}, 3 \mathrm{H}, \mathrm{NCH}_{3}\right)$, $6.37\left(\mathrm{q}, J=7.6 \mathrm{~Hz}, 1 \mathrm{H},=\mathrm{CHCH}_{3}\right), 7.04(\mathrm{~s}, 1 \mathrm{H}$, $\mathrm{NCH}=), 7.13-7.18(\mathrm{~m}, 1 \mathrm{H}, \mathrm{ArH}), 7.25-7.42(\mathrm{~m}, 4 \mathrm{H}$, ArH $), 7.56-7.61(\mathrm{~m}, 1 \mathrm{H}, \mathrm{ArH}), 7.77-7.80(\mathrm{~m}, 1 \mathrm{H}$, $\mathrm{ArH})$. 
${ }^{13} \mathrm{C} \mathrm{NMR}\left(75.4 \mathrm{MHz}, \mathrm{CDCl}_{3}\right): \delta=6.0\left(2 \times \mathrm{CH}_{2}\right), 9.7$ $(\mathrm{CH}), 15.7\left(\mathrm{CH}_{3}\right), 33.1\left(\mathrm{CH}_{3}\right), 109.0(\mathrm{C}), 109.3(\mathrm{CH})$, $119.5(\mathrm{CH}), 120.0(\mathrm{CH}), 121.0(\mathrm{CH}), 121.8(\mathrm{CH})$, $123.7(\mathrm{CH}), 126.8(\mathrm{CH}), 128.8(\mathrm{C}), 129.1(\mathrm{CH}), 130.2$ (C), $131.3(\mathrm{CH}), 133.0$ (C), 136.8 (C), $137.4(\mathrm{C})$, 138.4 (C), 140.8 (C), 142.9 (C).

MS (EI, $70 \mathrm{eV}): m / z(\%)=345\left(100, \mathrm{M}^{+}\right), 330(50)$, 295 (29).

HRMS-EI: $m / z$ [M] ${ }^{+}$calcd for $\mathrm{C}_{23} \mathrm{H}_{20} \mathrm{ClN}$ : 345.1284; found: 345.1293 .

$\mathbf{5 j}$, yellow solid; yield: $47 \mathrm{mg}(35 \%) ; R_{f}=0.25$ (hexane-Et ${ }_{2} \mathrm{O}, 15: 1$ ); $\mathrm{mp}=215-217^{\circ} \mathrm{C}$.

${ }^{1} \mathrm{H} \mathrm{NMR}\left(300 \mathrm{MHz}, \mathrm{CDCl}_{3}\right): \delta=2.21\left(\mathrm{~s}, 3 \mathrm{H}, \mathrm{CCH}_{3}\right)$, $3.88\left(\mathrm{~s}, 3 \mathrm{H}, \mathrm{NCH}_{3}\right), 5.66(\mathrm{~s}, 1 \mathrm{H}, H \mathrm{HC}=\mathrm{C}), 6.07(\mathrm{~s}$, $1 \mathrm{H}, \mathrm{H} H \mathrm{C}=\mathrm{C}), 7.05(\mathrm{~s}, 1 \mathrm{H}, \mathrm{NCH}=), 7.12-7.18(\mathrm{~m}, 1 \mathrm{H}$, ArH), 7.23-7.42 (m, 5H, ArH), 7.55-7.59 (m, 1H, ArH), 7.62-7.69 (m, 1H, ArH).

${ }^{13} \mathrm{C} \mathrm{NMR}\left(75.4 \mathrm{MHz}, \mathrm{CDCl}_{3}\right): \delta=12.2\left(\mathrm{CH}_{3}\right), 33.1$ $\left(\mathrm{CH}_{3}\right), 108.9(\mathrm{C}), 109.4(\mathrm{CH}), 111.7\left(\mathrm{CH}_{2}\right), 118.4$ $(\mathrm{CH}), 119.4(\mathrm{CH}), 119.5(\mathrm{CH}), 121.1(\mathrm{CH}), 121.9$ $(\mathrm{CH}), 125.1(\mathrm{CH}), 128.2(\mathrm{CH}), 128.3(\mathrm{C}), 128.8(\mathrm{CH})$, 130.6 (C), 136.4 (C), 137.1 (C), 138.5 (C), 144.7 (C), 148.0 (C).

MS (EI, $70 \mathrm{eV}): m / z(\%)=271\left(100, \mathrm{M}^{+}\right), 270(75)$, 256 (36), 127 (19).

HRMS-EI: $m / z[\mathrm{M}]^{+}$calcd for $\mathrm{C}_{20} \mathrm{H}_{17} \mathrm{~N}: 271.1361$; found: 271.1352 .

4j, white solid; yield: $26 \mathrm{mg}(18 \%) ; R_{f}=0.25$ (hexane- $\mathrm{Et}_{2} \mathrm{O}, 1: 2$ ); $\mathrm{mp}=83-85^{\circ} \mathrm{C}$.

${ }^{1} \mathrm{H}$ NMR $\left(300 \mathrm{MHz}, \mathrm{CDCl}_{3}\right): \delta=2.23(\mathrm{~d}, J=1.9 \mathrm{~Hz}$, $\left.3 \mathrm{H}, \mathrm{CH}_{3}\right), 3.74(\mathrm{dd}, J=10.8,5.8 \mathrm{~Hz}, 1 \mathrm{H}, \mathrm{CH} H \mathrm{OH})$, $3.85(\mathrm{~s}, 3 \mathrm{H} \mathrm{NCH}), 4.00(\mathrm{dd}, J=10.8,4.7 \mathrm{~Hz}, 1 \mathrm{H}$, $\mathrm{CH} \mathrm{HOH}$ ), 4.20 (bs, $1 \mathrm{H}$ min, $\mathrm{CHCHOH}), 7.13(\mathrm{~s}, 1 \mathrm{H}$, $\mathrm{NCH}=), 7.14-7.20(\mathrm{~m}, 1 \mathrm{H}, \mathrm{ArH}), 7.21-7.39(\mathrm{~m}, 5 \mathrm{H}$, ArH), 7.56-7.59 (m, 1H, ArH), 7.66-7.70 (m, 1H, $\mathrm{ArH})$. The signal corresponding to the $\mathrm{OH}$ is not observed.

${ }^{13} \mathrm{C}$ NMR $\left(75.4 \mathrm{MHz}, \mathrm{CDCl}_{3}\right): \delta=12.3\left(\mathrm{CH}_{3}\right), 33.1$ $\left(\mathrm{CH}_{3}\right), 54.5(\mathrm{CH}), 64.1\left(\mathrm{CH}_{2}\right), 109.7(\mathrm{CH}), 110.8(\mathrm{C})$, $119.0(\mathrm{CH}), 120.0(\mathrm{CH}), 120.6(\mathrm{CH}), 122.2(\mathrm{CH})$, $123.0(\mathrm{CH}), 124.6(\mathrm{CH}), 127.3(\mathrm{CH}), 127.4(\mathrm{C}), 128.4$ (CH), 134.8 (C), 136.2 (C), 137.2 (C), 144.2 (C), 147.4 (C).

MS (EI, $70 \mathrm{eV}): m / z(\%)=289\left(65, \mathrm{M}^{+}\right), 258(100)$, 205 (18).

HRMS-EI: $m / z$ [M] ${ }^{+}$calcd for $\mathrm{C}_{20} \mathrm{H}_{19} \mathrm{NO}: 289.1467$; found: 289.1462 .

5k, orange solid; yield: $42 \mathrm{mg}(30 \%) ; R_{f}=0.21$ (hexane- $\left.\mathrm{Et}_{2} \mathrm{O}, 15: 1\right) ; \mathrm{mp}=70^{\circ} \mathrm{C}$ (decomposition).

${ }^{1} \mathrm{H} \mathrm{NMR}\left(300 \mathrm{MHz}, \mathrm{CDCl}_{3}\right): \delta=2.17\left(\mathrm{~s}, 3 \mathrm{H}, \mathrm{CCH}_{3}\right)$, $3.86\left(\mathrm{~s}, 3 \mathrm{H}, \mathrm{NCH}_{3}\right), 5.66(\mathrm{~s}, 1 \mathrm{H}, H \mathrm{HC}=\mathrm{C}), 5.92(\mathrm{~s}$, $1 \mathrm{H}, \mathrm{H} H \mathrm{C}=\mathrm{C}), 6.99(\mathrm{~s}, 1 \mathrm{H}, \mathrm{NCH}=), 7.11-7.16(\mathrm{~m}, 2 \mathrm{H}$,
ArH), 7.19-7.22 (m, 1H, ArH), 7.23-7.30 (m, 1H, ArH), 7.35-7.39 (m, 1H, ArH), 7.56-7.60 (m, 1H, ArH).

${ }^{13} \mathrm{C}$ NMR $\left(75.4 \mathrm{MHz}, \mathrm{CDCl}_{3}\right): \delta=13.8\left(\mathrm{CH}_{3}\right), 33.0$ $\left(\mathrm{CH}_{3}\right), 109.0(\mathrm{C}), 109.4(\mathrm{CH}), 115.2\left(\mathrm{CH}_{2}\right), 119.5$ $(\mathrm{CH}), 119.7(\mathrm{CH}), 121.0(\mathrm{CH}), 121.9(\mathrm{CH}), 124.2$ $(\mathrm{CH}), 128.3(\mathrm{C}), 128.7(\mathrm{CH}), 129.1(\mathrm{C}), 134.0(\mathrm{C})$, 137.0 (C), 140.2 (C), 144.9 (C), 149.0 (C).

MS (EI, $70 \mathrm{eV}): m / z(\%)=277\left(100, \mathrm{M}^{+}\right), 276(51)$, 262 (30), 261 (31).

HRMS-EI: $m / z[\mathrm{M}]^{+}$calcd for $\mathrm{C}_{18} \mathrm{H}_{15} \mathrm{NS}: 277.0925$; found: 277. 0927.

5l, isolated as a $\sim 5: 1$ mixture $E / Z$; yellow solid; yield: $78 \mathrm{mg}(50 \%) ; R_{f}=0.30$ (hexane- $\mathrm{Et}_{2} \mathrm{O}, 15: 1$ ); $\mathrm{mp}=$ $146-148{ }^{\circ} \mathrm{C}$. NMR data of the major isomer are provided.

${ }^{1} \mathrm{H}$ NMR $\left(300 \mathrm{MHz}, \mathrm{CDCl}_{3}\right): \delta=1.33(\mathrm{~d}, J=7.1 \mathrm{~Hz}$, $\left.3 \mathrm{H}, \quad \mathrm{CH}_{3} \mathrm{CHCH}_{3}\right), 1.39(\mathrm{~d}, J=7.1 \mathrm{~Hz}, 3 \mathrm{H}$, $\left.\mathrm{CH}_{3} \mathrm{CHCH}_{3}\right), 2.31\left(\mathrm{~d}, J=7.6 \mathrm{~Hz}, 3 \mathrm{H}, \mathrm{CH}=\mathrm{CH}_{3}\right), 3.15$ (sept, $\left.J=7.1 \mathrm{~Hz}, 1 \mathrm{H}, \mathrm{CH}_{3} \mathrm{CHCH}_{3}\right), 3.88(\mathrm{~s}, 3 \mathrm{H}$, $\left.\mathrm{NCH}_{3}\right), 6.22\left(\mathrm{q}, J=7.6 \mathrm{~Hz}, 1 \mathrm{H}, \mathrm{CH}=\mathrm{CH}_{3}\right), 6.97(\mathrm{~s}$, $1 \mathrm{H}, \mathrm{NCH}=), 7.11-7.17(\mathrm{~m}, 1 \mathrm{H}, \mathrm{ArH}), 7.22-7.36(\mathrm{~m}$, $3 \mathrm{H}, \mathrm{ArH}), 7.37-7.43(\mathrm{~m}, 1 \mathrm{H}, \mathrm{ArH}), 7.49-7.55(\mathrm{~m}, 1 \mathrm{H}$ min, ArH), 7.58-7.65 (m, 1H, ArH), 7.87 (d, $J=7.5$ $\mathrm{Hz}, 1 \mathrm{H}, \mathrm{ArH})$.

${ }^{13} \mathrm{C}$ NMR $\left(75.4 \mathrm{MHz}, \mathrm{CDCl}_{3}\right): \delta=15.6\left(\mathrm{CH}_{3}\right), 21.5$ $\left(\mathrm{CH}_{3}\right), 21.8\left(\mathrm{CH}_{3}\right), 27.8(\mathrm{CH}), 33.0\left(\mathrm{CH}_{3}\right), 109.3$ $(\mathrm{CH}), 109.5(\mathrm{C}), 119.4(\mathrm{CH}), 120.7(\mathrm{CH}), 120.8(\mathrm{CH})$, $121.8(\mathrm{CH}), 123.8(\mathrm{CH}), 124.4(\mathrm{CH}), 126.8(\mathrm{CH})$, $128.8(\mathrm{CH}), 129.4(\mathrm{C}), 130.2(\mathrm{CH}), 130.8(\mathrm{C}), 136.7$ (C), 136.9 (C), 142.0 (C), 143.2 (C), 145.2 (C).

MS (EI, $70 \mathrm{eV}): \mathrm{m} / z(\%)=313\left(100, \mathrm{M}^{+}\right), 298(68)$, 270 (42), 202 (35).

HRMS-EI: $m / z[\mathrm{M}]^{+}$calcd for $\mathrm{C}_{23} \mathrm{H}_{23} \mathrm{~N}$ : 313.1830; found: 313.1821 .

4l, isolated as a $\sim 6: 1$ mixture of diastereoisomers, white solid; yield: $51 \mathrm{mg}(31 \%) ; \quad R_{f}=0.24$ (hexane- $\left.\mathrm{Et}_{2} \mathrm{O}, 1: 3\right) ; \mathrm{mp}=66-68^{\circ} \mathrm{C}$. NMR data of the major isomer are provided.

${ }^{1} \mathrm{H} \mathrm{NMR}\left(300 \mathrm{MHz}, \mathrm{CDCl}_{3}\right): \delta=1.06(\mathrm{~d}, J=6.4 \mathrm{~Hz}$, $3 \mathrm{H}, \mathrm{CHOHCHCH}), 1.26(\mathrm{~d}, J=7.1 \mathrm{~Hz}, 3 \mathrm{H}$, $\left.\mathrm{CH}_{3} \mathrm{CHCH}_{3}\right), 1.44(\mathrm{bs}, 1 \mathrm{H}, \mathrm{OH}), 1.53(\mathrm{~d}, J=7.1 \mathrm{~Hz}$, $3 \mathrm{H}, \mathrm{CH}_{3} \mathrm{CHCH}_{3}$ ), 3.30 (sept, $J=7.1 \mathrm{~Hz}, 1 \mathrm{H}$, $\left.\mathrm{CH}_{3} \mathrm{CHCH}_{3}\right), 3.86\left(\mathrm{~s}, 3 \mathrm{H}, \mathrm{NCH}_{3}\right), 4.19(\mathrm{~d}, J=3.3 \mathrm{~Hz}$, $1 \mathrm{H}, \mathrm{CHCHOH}), 4.23-4.30(\mathrm{~m}, 1 \mathrm{H}, \mathrm{CHOH}), 7.07(\mathrm{~s}$, $1 \mathrm{H}, \mathrm{NCH}=), 7.15-7.25(\mathrm{~m}, 2 \mathrm{H}, \mathrm{ArH}), 7.28-7.41(\mathrm{~m}$, $3 \mathrm{H}, \mathrm{ArH}), 7.51-7.55$ (m, 1H, ArH), 7.61-7.68 (m, 2H $\min , \mathrm{ArH})$.

${ }^{13} \mathrm{C}$ NMR $\left(75.4 \mathrm{MHz}, \mathrm{CDCl}_{3}\right): \delta=19.4\left(\mathrm{CH}_{3}\right), 21.2$ $\left(\mathrm{CH}_{3}\right), 21.7\left(\mathrm{CH}_{3}\right), 27.6(\mathrm{CH}), 33.1\left(\mathrm{CH}_{3}\right), 58.7(\mathrm{CH})$, $69.6(\mathrm{CH}), 109.7(\mathrm{CH}), 111.4(\mathrm{C}), 120.0(\mathrm{CH}), 120.3$ $(\mathrm{CH}), 121.5(\mathrm{CH}), 122.3(\mathrm{CH}), 123.8(\mathrm{CH}), 124.2$ $(\mathrm{CH}), 126.7(\mathrm{CH}), 127.7(\mathrm{C}), 128.1(\mathrm{CH}), 134.7(\mathrm{C})$, 137.2 (C), 144.3 (C), 144.9 (C), 146.7 (C). 
MS (EI, $70 \mathrm{eV}): \mathrm{m} / \mathrm{z}(\%)=331\left(96, \mathrm{M}^{+}\right), 287(60)$, 272 (91), 244 (100).

HRMS-EI: $\mathrm{m} / z$ [M] $]^{+}$calcd for $\mathrm{C}_{23} \mathrm{H}_{25} \mathrm{NO}: 331.1936$; found: 331.1935 .

Supporting Information for this article is available online at http://www.thiemeconnect.com/ejournals/toc/synthesis.

\section{Acknowledgment}

We acknowledge MICINN (CTQ2010-15358 and CTQ200909949) and Junta de Castilla y León (BU021 A09 and GR-172) for financial support. We are also grateful to MEC (FPU predoctoral fellowships to E. A. and D. M., "Ramón y Cajal" contract to M. A. F. R. and "Juan de la Cierva" contract to P. G. G.

\section{References}

(1) New address: Departamento de Química Orgánica. Facultad de Ciencias. Universidad de Granada. 18071Granada (Spain).

(2) (a) Tietze, L. F. Chem. Rev. 1996, 96, 115. (b) Tietze, L. F.; Brasche, G.; Gericke, K. Domino Reactions in Organic Synthesis; Wiley-VCH: Weinheim, 2006, 672. (c) Grossmann, A.; Enders, D. Angew. Chem., Int. Ed. 2012, 51,314 .

(3) (a ) Mayer, S. F.; Kroutil, W.; Faber, K. Chem. Soc. Rev. 2001, 30, 332. (b) McCarroll, A. J.; Walton, J. C. Angew. Chem., Int. Ed. 2001, 40, 2224. (c) Nicolaou, K. C.; Edmonds, D. J.; Bulger, P. G. Angew. Chem., Int. Ed. 2006, 45, 7134 .

(4) (a) Fogg, D. E.; dos Santos, E. N. Coord. Chem. Rev. 2004, 248, 2365. (b) Shindoh, N.; Takemoto, Y.; Takasu, K. Chem. Eur. J. 2009, 15, 12168.

(5) Bruggink, A.; Schoevaart, R.; Kieboom, T., Org. Proc. Res. Dev. 2003, 7, 622.

(6) For a recent review, see: Vlaar T.; Ruijter, E.; Orru, R. V. A. Adv. Synth. Catal. 2011, 353, 809.

(7) For recent reviews, see: (a) Nolan, S. P. Acc. Chem. Res. 2011, 44, 91. (b) Corma, A.; Leyva-Pérez, A.; Sabater, M. J. Chem. Rev. 2011, 111, 1657. (c) Krause, N.; Winter, C. Chem. Rev. 2011, 111, 1994. (d) Wegner, H. A.; Auzias, M. Angew. Chem., Int. Ed. 2011, 50, 8236. (e) Hopkinson, M. N.; Gee, A. D.; Gouverneur, V. Chem. Eur. J. 2011, 17, 8242.

(8) For recent examples of $\mathrm{Au}(\mathrm{I})$-catalyzed tandem reaction initiated by migration of propargyl esters, see: (a) Zhang, D.-H.; Yao, L.-F.; Wei, Y.; Shi, M. Angew. Chem., Int. Ed. 2011, 50, 2583. (b) Lebœuf, D.; Simonneau, A.; Aubert, C.; Malacria, M.; Gandon, V.; Fensterbank, L. Angew. Chem. Int. Ed. 2011, 50, 6868 (c) Rao, W.; Susanti, D.; Chan, P. W. H. J. Am. Chem. Soc. 2011, 133, 15248.

(9) See, for example: (a) Nakano, T.; Takewaki, K.; Yade, T.; Okamoto, Y. J. Am. Chem. Soc. 2001, 123, 9182. (b) Cappelli, A.; Mohr, G. P.; Anzini, M.; Vomero, S.; Donati, A.; Casolaro, M.; Mendichi, R.; Giorgi, G.; Makovec, F. J. Org. Chem. 2003, 68, 9473.

(10) Alt, H. G.; Köppl, A. Chem. Rev. 2000, 100, 1205.

(11) (a) Wang, S.; Yang, Q.; Mak, T. C. W.; Xie, Z. Organometallics 2000, 19, 334. (b) Kovalenko, S. V.; Peabody, S.; Manoharan, M.; Clark, R. J.; Alabugin, I. V. Org. Lett. 2004, 6, 2457.

(12) See, for instance: Clegg, N. J.; Paruthiyil, S.; Leitman, D. C.; Scanlan, T. S. J. J. Med. Chem. 2005, 48, 5989.
(13) For selected recent benzofulvene syntheses from monocyclic precursors, see: (a) Lee, C.-Y.; Wu, M.-J. Eur. J. Org. Chem. 2007, 3463. (b) Bucher, G.; Mahajan, A. A.; Schmittel, M. J. Org. Chem. 2008, 73, 8815. (c) Cordier, P.; Aubert, C.; Malacria, M.; Lacôte, E.; Gandon, V. Angew. Chem., Int. Ed. 2009, 48, 8757. (d) Jeffrey, J. L.; Sarpong, R. Tetrahedron Lett. 2009, 50, 1969. (e) Ye, S.; Gao, K.; Zhou, H.; Yang, X.; Wu, J. Chem. Commun. 2009, 5406. (f) Bryan, C. S.; Lautens, M. Org. Lett. 2010, 12, 2754. (g) Ye, S.; Yang, X.; Wu, J. Chem. Commun. 2010, 46, 2950. (h) Tsuchikama, K.; Kasagawa, M.; Endo, K.; Shibata, T. Synlett 2010, 97. (i) Chinnagolla, R. K.; Jeganmohan, M. Eur. J. Org. Chem. 2012, 417.

(14) (a) Sanz, R.; Miguel, D.; Álvarez-Gutiérrez, J. M.; Rodríguez, F. Synlett 2008, 975. (b) Sanz, R.; Miguel, D.; Martínez, A.; Gohain, M.; García-García, P.; Fernández-Rodríguez, M. A.; Álvarez, E.; Rodríguez, F. Eur. J. Org. Chem. 2010, 7027.

(15) (a) Sanz, R.; Miguel, D.; Rodríguez, F. Angew. Chem., Int. Ed. 2008, 47, 7354. (b) Sanz, R.; Miguel, D.; Gohain, M.; García-García, P.; Fernández-Rodríguez, M. A.; González-Pérez, A.; Nieto-Faza, O.; de Lera, A. R.; Rodríguez, F. Chem. Eur. J. 2010, 16, 9818.

(16) The selectivity of this tandem reaction (1,2-migration followed by aura-iso-Nazarov cyclization) could be switched by the proper choice of catalyst and reaction conditions allowing the isolation of 2-(inden-3yl)indenes derived from an aura-Nazarov cyclization. See: Álvarez, E.; Miguel, D.; García-García, P.; Fernández-Rodríguez, M. A.; Rodríguez, F.; Sanz, R. Beilstein J. Org. Chem. 2011, 7, 786.

(17) (a) Marion, N.; Nolan, S. P. Angew. Chem., Int. Ed. 2007, 46, 2750. (b) Marco-Contelles, J.; Soriano, E. Chem. Eur. J. 2007, 13, 1350. (c) Wang, S.; Zhang, G.; Zhang, L. Synlett 2010, 692.

(18) (a) Martínez, A.; García-García, P.; FernándezRodríguez, M. A.; Rodríguez, F.; Sanz, R. Angew. Chem., Int. Ed. 2010, 49, 4633. (b) Sanz, R.; Martínez, A.; García-García, P.; Fernández-Rodríguez, M. A.; Rashid, M. A.; Rodríguez, F. Chem. Commun. 2010, 46, 7427.

(19) Mézailles, N.; Ricard, L.; Gagosz, F. Org. Lett. 2005, 7, 4133.

(20) The stereochemistry of the double bond was assigned by NOESY experiments. See Supporting Information.

(21) CCDC 869753 (5c) contains the supplementary crystallographic data for this paper. These data can be obtained free of charge from The Cambridge Crystallographic Data Centre via www.ccdc.cam.ac.uk/data_request/cif 
Please place the graphical abstract and short title of the article here. The short title will be used as a running header.

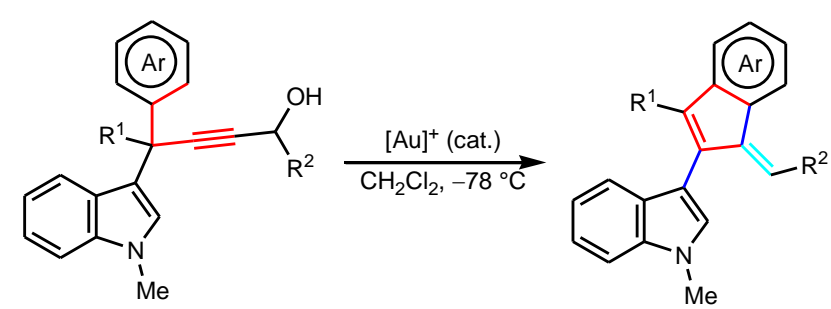

Benzofulvenes by a Tandem Reaction

\section{Manuscript submission checklist}

- Statement of significance of work.

- Full mailing address, telephone, and fax numbers and e-mail address of the corresponding author.

- Graphical abstract.

- 5 key words.

- Original Word file.

- Word file saved as a PDF file.

- Original graphics files.

Send all the materials on this list to the Special Topics Editor. 